\title{
Dos especies nuevas de Borreria (Rubiaceae), sinopsis y clave de las especies para Bahia, Brasil
}

Elsa Leonor Cabral ${ }^{1,2}$, Laila Mabel Miguel ${ }^{1}$ y Roberto Manuel Salas ${ }^{1}$

Recebido em 29/07/2010. Aceito em 28/02/2011

\section{RESUMEN}

(Dos especies nuevas de Borreria G. Mey. (Rubiaceae), sinopsis y clave de las especies para Bahia, Brasil). El presente trabajo trata del estudio taxonómico del género Borreria (Rubiaceae) para el estado de Bahia, siendo registradas 29 especies. La mayor parte de estas especies habitan en los biomas del cerrado y la mata atlántica. Se describen e ilustran dos especies nuevas, Borreria catolensis E.L. Cabral \& L.M. Miguel y B. diamantinae R.M. Salas \& E.L. Cabral. Se realiza la nueva combinación, Borreria schumannii (Standl. ex Bacigalupo) E.L. Cabral \& Sobrado. Se selecciona neotipo para B. virgata. Se elige epitipo para B. scabiosoides y se designa lectotipos para B. eryngioides y $B$. humifusa. Se presenta clave de identificación, comentarios sobre distribución geográfica y hábitat, se incluyen mapas y un cuadro de distribución de las especies por bioma. Se presenta por primera vez ilustraciones de B. crispata, B. cupularis, B. humifusa, B. virgata y B. wunschmannii. Se discute las identidades de B. ocymoides y B. prostrata, la primera de las cuales es también ilustrada. Se rehabilitan a B. virgata y B. spinosa. Se incluye además dos figuras con 20 especies.

Palabras clave: nomenclatura, Spermacoceae, taxonomía

\begin{abstract}
(Two new species of Borreria (Rubiaceae), synopsis and key to distinguish the species from Bahia, Brazil). This work deals with a taxonomic study of the genus Borreria (Rubiaceae) for the state of Bahia, 29 species are recorded. Most of these species are distributed in the cerrado and Atlantic forest biomes. Two new species are described and illustrated, Borreria catolensis E.L. Cabral \& L.M. Miguel and Borreria diamantinae R.M. Salas \& E.L. Cabral. The new combination Borreria schumannii (Standl. ex Bacigalupo) E.L. Cabral \& Sobrado is proposed. Neotype is designated for B. virgata. Epitype is designated for B. scabiosoides and lectotypes are designated for B. eryngioides and B. humifusa. A key for identification, comments on geographic distribution and habitats are provided, maps and a table with the species distribution for biomes are also included. Borreria crispata, B. cupularis, B. humifusa, B. virgata and B. wunschmannii are illustrated for the first time. The identities of B. ocymoides and B. prostrata are discussed; the first species is also illustrated. Borreria virgata and B. spinosa are reinstated. Two figures with representative photographs of 20 species are included.
\end{abstract}

Key words: nomenclature, Spermacoceae, taxonomy

\section{Introducción}

Borreria G. Mey. pertenece a la tribu Spermacoceae, donde la delimitación de los géneros representa el principal y más actual problema, debido en gran parte a la similitud morfológica entre los taxones y a las opiniones opuestas entre los autores. Clásicamente, el tipo de dehiscencia de la cápsula ha jugado un rol muy importante en la delimitación de géneros; mientras que recientemente los límites intergenéricos han sido claramente modificados en base a caracteres moleculares, ampliando el campo de discusión. En este sentido, Borreria es un género que presenta esta problemática. Autores del Neotrópico mantuvieron a este género separado del más afín Spermacoce L., delimitándolos en base a caracteres polínicos y carpológicos (Bacigalupo 1972; Steyermark 1972, 1974; Bacigalupo \& Cabral 1996, 2007; Cabral \& Bacigalupo 1996; Taylor

\footnotetext{
1 Universidad Nacional del Nordeste, FACENA, Instituto de Botánica del Nordeste, CONICET, Corrientes, Argentina

2 Autor para correspondencia: ecabral@agr.unne.edu.ar
} 
\& Steyermark 2004; Bacigalupo et al. 2010; Cabral et al. 2010), siendo además parcialmente apoyados por datos moleculares (Dessein 2003). Esta postura es rechazada por la mayoría de los investigadores que estudian el $\mathrm{Pa}$ leotrópico, quienes consideran solamente a Spermacoce s.l., incluyendo a Borreria (Verdcourt 1976; Dessein 2003) y han propuesto innumerables nuevos nombres y nuevas combinaciones, acrecentando la dificultad en la determinación de las especies. Basados en este concepto transfirieron numerosas especies a Spermacoce y en algunos casos sin reparar en la pertenencia genérica.

Por lo expuesto, se optó por el momento, estudiar a estas especies como Borreria tratando de sortear la creación de nuevos binomios a fin de evitar mayor confusión. Esta posición se fundamenta en que los trabajos actuales de filogenia no son aún concluyentes e incluyen un número reducido de especies que no representa a la tribu en la flora Neotropical (matrices entre 16 y 60 especies de ca. 230).

En este trabajo se han incluido cuatro especies que fueron descritas originalmente bajo Diodia L. en sentido amplio, siguiendo los cambios propuestos por Bacigalupo \& Cabral (1996, 1998, 1999): Borreria multiflora (DC.) Bacigalupo \& E.L. Cabral, B. palustris (Cham. \& Schltdl.) Bacigalupo \& E.L. Cabral, B. pulchristipula (Bremek.) Bacigalupo \& E.L. Cabral y Borreria schumannii (Standl. ex Bacigalupo) E.L. Cabral \& Sobrado (una nueva combinación).

Como parte del análisis de colecciones recientes y como una contribución al estudio taxonómico del género Borreria en Bahia, se presenta una sinopsis de las 29 especies que habitan en el estado y una clave para diferenciarlas. Se describen e ilustran dos especies nuevas y se realiza una nueva combinación. Se designa neotipo para el nombre de B. virgata Cham. \& Schltdl., se elige epitipo para $B$. scabiosoides Cham. \& Schltdl. y se selecciona lectotipos para B. eryngioides Cham. \& Schltdl. y B. humifusa Mart. Además, se reestablecen B. virgata Cham. \& Schltdl. y $B$. spinosa (L.) Cham. \& Schltdl. y se discute las identidads de B. ocymoides (Burm. f.) DC. Se confirman siete primeros registros para este estado.

\section{Materiales y métodos}

El presente trabajo está basado en métodos convencionales de taxonomía. Se examinó material proveniente de los siguientes herbarios: ALCB, BM, B, BR, B-W, CEPEC, CTES, ESA, F, HAL, HUEFS, HST, G, G-DC, IAN, K, LE, LIL, MBM, MG, MO, NY, P, R, SI, SP, SPF, U, UB, UC, UESC, ULM, US, USP, W (Holmgren et al. 1990). Los datos expuestos sobre la fitogeografía de las especies de Borreria fueron tomados de observaciones de campo en viajes realizados en Agosto 2008, Abril-Mayo 2009, Mayo-Junio 2010 y sobre todo de abundante material de herbario. Las formaciones vegetales fueron delimitadas principalmente con los datos más comunes de las etiquetas en su definición más general (cerrado s.l., campos rupestres como un ambiente diferente de caatinga), comparados con los datos expuestos por Giulietti et al. (2006) y los datos de distribución se muestran en mapas.

Se incluyen sinónimos sólo en las especies donde se han resuelto conflictos taxonómicos: Borreria humifusa, $B$. latifolia, B.ocymoides, B. prostrata, B. spinosa, B. tenella, B. virgata y $B$. wunschmannii; y además en aquellas que estuvieron involucradas con el género Diodia (B. multiflora, $B$. palustris, B. pulchristipula y B. schmannii).

\section{Tratamiento taxonómico}

Borreria G. Mey., Prim. Fl. Esseq.: 79, pl. 1. 1818.

Especie tipo: Borreria suaveolens G. Mey.

Fig. 1-10

Hierbas o sufrútices, apoyantes, erectos o decumbentes. Flores pequeñas en glomérulos axilares y/o terminales. Cáliz persistente 4(2)-partido. Corola 4-lobada. Estambres 4, exertos o inclusos. Estilo de ápice bífido o capitadobilobado. Fruto capsular, septicida, mericarpos dehiscentes unidos por su base o con mericarpos indehiscentes con una línea media longitudinal de dehiscencia preformada en la cara ventral. Semillas de superficie reticulada-foveolada, a veces surcadas transversalmente, con surco ventral cubierto por estrofíolo, rara vez con elaiosomas.

El género es pantropical, con ca. 100 especies americanas cuyo principal centro de concentración es Brasil, donde crecen 63 especies, 31 de las cuales son endémicas (Cabral \& Salas 2010). En Bahia, habitan 29 especies, siendo tres de ellas exclusivas para el estado, Borreria bahiana, $B$. catolensis y $B$. diamantinae; siendo ocho las más comunes: B. capitata, B. crispata, B. latifolia, B. ocymifolia, $B$. pulchristipula, B. scabiosoides, B. verticillata y B. virgata. Crecen en suelos lateríticos, arenosos o con afloramientos rocosos. En Bahia, habitan en los tres grandes biomas del estado (Giulietti et al. 2006). En el Cerrado (CER), viven 15 especies, siete aparentemente exclusivas para este bioma, mientras que en los Campos Rupestres (CP) crecen solo ocho especies, tres de las cuales son endémicas: $B$. bahiana, B. catolensis y B. diamantianae. En las Caatingas (CAA), habitan ocho especies, que comparten con otros biomas. En la Mata Atlántica (MA), se encontraron 16 especies, de las cuales cinco aparentemente exclusivas de este bioma, $B$. humifusa, $B$. palustris, $B$. oligodonta, $B$. schumannii y $B$. virgata, siendo $B$. oligodonta mencionada por primera vez fuera de la Amazonia. Entre las especies bahianas, existen 10 que pueden ser consideradas malezas (MAL), destacándose B. capitata, B. latifolia, B. spinosa y $B$. verticillata como especies altamente invasoras. Con respecto a $B$. paraensis, no se conocen datos precisos (DI: datos insuficientes), sin embargo en una de las etiquetas la menciona en áreas cercanas al mar y en una localidad llamada "Cabulla" (Cabuya, Salvador), dato que se utilizó para ubicarla tentativamente en el mapa (Tab. 1). 
1. Planta postrada sólo con eje florífero erecto, sin rizoma ni xilopodio

2. Hojas heteromorfas; glomérulo apical largamente pedunculado, con 4 brácteas cordiformes amplexifloras; semilla no ruminada, sin elaiosoma

9. B. humifusa

2. Hojas isomorfas; glomérulo apical y subapical cortamente pedunculado, con 2-4 brácteas elípticas no amplexifloras; semilla ruminada, con elaiosoma

1. Planta erecta, decumbente o escandente, a veces con rizoma o con xilopodio

3. Hojas filiformes, lineares o linear-lanceoladas, 0,3-2(-10) mm lat.

4. Tallos simples desde la base que terminan en 1 o 2 ejes floríferos; hojas aciculares, 0,5-1 mm lat., glabras, de sección subcircular, sin braquiblastos en las axilas; glomérulo apical con 2(3) brácteas

4. B. catolensis

4. Tallos con ramas laterales desde la base que terminan en 1-5 ejes floríferos; hojas lineares o linear-lanceoladas, 0,5$10 \mathrm{~mm}$ lat., glabras o pubescentes, de sección aplanada, generalmente con braquiblastos en las axilas; glomérulos 1-3 por eje florífero, con 2-8 brácteas

5. Planta pubescente, pelos adpresos retrorsos, rojizos; vaina estipular pubescente con 6 o 7 lacinias rojizas pilosas o glabrescentes.

29. B. wunschmannii

5. Planta glabra o pubérula; vaina estipular pubérula o pilosa con 3 o 4(-6) lacinias glabras no rojizas

6. Planta robusta; glomérulo 10-14 mm lat., 6-12 brácteas iguales o más cortas que el glomérulo; semilla no ruminada, con elaiosoma

5. B. crispata

6. Planta grácil; glomérulo 2,5-7 mm lat., 2(-4) brácteas más largas que el glomérulo; semilla ruminada, sin elaiosoma

17. B.paraensis

3. Hojas elípticas, elíptico-lanceoladas, ovadas de 0,7-35 mm lat.

7. Estambres y estilo inclusos

8. Arbusto de 1-2,5 $\mathrm{m}$ alt.; segmentos del cáliz 5-8,6 mm long., notablemente reflexos.

21. B. reflexa

8. Hierba o sufrútice 0,25-0,60 m alt., segmentos del cáliz 0,5-2,5 mm long., no reflexos

9. Ejes no ramificados en pseudodicotomía, alados y solo con pelos largos en ángulos; hojas ovadas, 5-12 mm lat.; cáliz 2-partido.

14. B. ocymoides

9. Ejes floríferos distalmente ramificados en pseudodicotomía, ápteros, pubescentes; hojas lineares, oblongo-lanceoladas o elíptico-lanceoladas de 1,5-5,5 mm lat.; cáliz 4 partido

10. Corola hasta 1,5 mm long., igual o más corta que el cáliz, superficie interna con escasos pelos sobre los lóbulos; disco 2-lobado; semilla con notables fóveas hexagonales

19. B. prostrata

10. Corola hasta 3,5 mm long., igual o más larga que el cáliz, superficie interna con pelos sobre los lóbulos o con pelos dispersos sobre el tubo; disco entero; semilla con fóveas menores inconspicuas

8. B. eryngioides

7. Estambres y estilo exertos

11. Glomérulos 8-25 axilares, desde la base del eje floral; brácteas iguales a lo largo del eje o disminuyendo de tamaño hacia el ápice

12. Estigma capitado-bilobado; fruto separado en 2 mericarpos, uno indehiscente y otro parcialmente dehiscente en la base, semillas con surcos transversales leves; glomérulos y brácteas disminuyendo en tamaño y madurez hacia el ápice de la inflorescencia.

13. B. ocymifolia

12. Estigma bífido; fruto separado en 2 mericarpos ambos indehiscentes o dehiscentes, semillas sin surcos transversales; glomérulos y brácteas de igual tamaño a lo largo de la inflorescencia (excepto B. multiflora)

13. Plantas de campos altos no rizomatosas, con tallos erectos o decumbentes, frecuentemente alados; frutos de mericarpos dehiscentes.

13. Plantas palustres, a veces rizomatosas, con tallos apoyantes o escandentes, no alados; frutos de mericarpos indehiscentes

14. Hojas con nervios poco visibles en el envés, membranáceas

20. B. pulchristipula

14. Hojas plegado-nervosas, coriáceas

15. Sufrútice erecto, rizomatozo; hojas 5-13 mm lat., escábridas, oscuras al secarse, de base truncada, subauriculada; borde de la vaina estipular truncado, a la misma altura del nacimiento de las hojas; glomérulo 5-8 mm lat.; segmentos del cáliz 1,2-1,4 mm long.; corola 3,5-4 mm long.; cápsula 3-3,5 mm long.; semilla 2-2,5 mm long., castañas

12. B. multiflora

15. Sufrútice escandente o semitrepador, no rizomatoso; hojas 11-30 mm lat., glabras o pubescentes, no escábridas, verde amarillentas al secarse, base atenuada; borde de la vaina estipular tubulosa, prolongado por encima del nacimiento de las hojas; glomérulo 8-13 mm lat.; segmentos del cáliz 2,5-4 mm long., corola 4,5-7 mm long.; cápsula 4-4,5 mm long., semilla 3-4 mm long., negras.

24. B. schumannii 
11. Glomérulos 1-5 axilares, a veces inflorescencias en ramificaciones distales en pleiocasios; brácteas iguales a lo largo del eje o aumentando de tamaño hacia el ápice

16. Tallos simples o escasamente ramificados

17. Planta palustre, con tallos fistulosos, verde-nigrescente al secarse; vaina estipular glabra con 3-5 lacinas; semilla con elaiosoma

17. Plantas de campos altos, con tallos macizos, verde-amarillentas o verdes al secarse; vaina estipular pilosa con 6-15 lacinias; semilla sin elaiosoma.

26. B. tenella

16. Tallo principal y secundarios ramificados

18. Cáliz 2-partido

19. Glomérulos hasta $22 \mathrm{~mm}$ lat.

20. Brácteas más cortas que el glomérulo; hipanto pubescente en la mitad superior; corola salmón; hojas oblanceoladas hasta $20 \mathrm{~mm}$ long., pubescentes.

1. B. bahiana

20. Brácteas más largas que el glomérulo; hipanto con anillo de pelos largos blanquecinos en el ápice; corola blanca; hojas elíptico-lanceoladas o elípticas hasta $65 \mathrm{~mm}$ long., glabras o glabrescentes con notorias papilas en la vena media.

25. B. spinosa

19. Glomérulos hasta $15 \mathrm{~mm}$ lat.

21. Tallos principales simples desde la base con ejes florales de ramificaciones distales; hojas pubescentes; semillas 2-2,5 mm long

11. B. marticrovettiana

21. Tallos principales y secundarios con numerosas ramificaciones laterales; hojas glabras o glabrescentes, con papilas sobre el nervio central en el envés; semillas 1,2-1,4 mm long.

22. Brácteas 2-3 veces más largas que el glomérulo; hojas elípticas de 20-60 × 2-12 mm.

27. B. verticillata

22. Brácteas iguales o más cortas que el glomérulo; hojas lineares de 6-12 x 0,5-2,5 mm

15. B. oligodonta

18. Cáliz 4-partido

23. Hojas plegado-nervosas, coriáceas o subcoriáceas, haz generalmente pubérula o pubescente

24. Hierba palustre, rizomatosa; inflorescencia tirsoide con inflorescencias parciales glomeriformes, en pleiocasio o en ejes simples; corola 3(2)-lobada; frutos con 2(3) mericarpos indehiscentes.

16. B.palustris

24. Sufrútice de campos altos; inflorescencias en ejes simples; corola 4-lobada; frutos con 2-mericarpos dehiscentes.

25. Planta con xilopodio, verde amarillenta al secarse; vaina estipular glabra o solo pubescente en el tercio superior, con 3 lacinias; corola de 5-12 mm long.; estigma bífido; semilla no ruminada.

18. B.poaya

25. Plantas sin xilopodio, verde o verde rojiza al secarse; vaina estipular pubérula, con 5-7 lacinias; corola 2,53,8(4,6) mm long.; estigma capitado-bilobado; semilla ruminada.

23. Hojas no plegado-nervosas, papiráceas o membranáceas, haz generalmente glabra

26. Segmentos del cáliz linear-lanceolados de 2-2,5 mm long.

27. Planta de 60-120 cm alt., escasamente ramificada, ramas primarias con 3-5 glomérulos; bractéolas notables, rojizas, mayores que el hipanto; semillas ruminadas con estrofiolo de mayor tamaño sobresaliendo en un extremo, sin elaiosoma...

.28. B. virgata

27. Planta de 25-40 cm alt., ramas primarias y secundarias con 1 o 2 glomérulos; bractéolas hialinas inconspicuas menores que el hipanto; semilla no ruminada, con estrofiolo de igual longitud, con elaiosoma cubriendo la mitad del surco ventral .

6. B. cupularis

26. Segmentos del cáliz ovados o triangulares de 0,4-1,8 mm long.

28. Hojas de 25-50 x 5-25 mm.; glomérulos 4-10 por rama florífera, 5-10 mm lat.; segmentos del cáliz triangulares con borde hírtulo; corola infundibuliforme

22. B. remota

28. Hojas de 40-140 x 10-45 mm.; glomérulos 1 o 2 por rama florífera, 10-30 mm lat.; segmentos del cáliz ovados, ciliados; corola ciatiforme

2. B. brownii

1. Borreria bahiana E.L. Cabral, Bol. Soc. Argent. Bot. 29(3-4): 227. 1993. Tipo: Brasil, Bahia, Barra da Estiva, camino a Ituaçu, Morro do Ouro y Morro da Torre, 1340'S, 41⒈'W, 23/X/1992, fl. fr., M.M. Arbo et al. 5723 (Holotipo SPF!; Isotipos CTES!, MBM!).

Fig. $5 \mathrm{~A}$ y 7

Esta especie es endémica de Bahia y su distribución está restringida a los campos rupestres de la Chapada Diamantina. Florece y fructifica de octubre a mayo.
Material seleccionado: BRASIL. Bahia: Barra da Estiva,

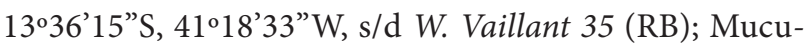
gê, Guiné, base da Serra do Esbarrancado, 1245'30"S, $41^{\circ} 30^{\prime} 43^{\prime \prime} \mathrm{W}, 4 / \mathrm{V} / 2009$, fl. fr., L.P. Queiroz et al. 14543 (CTES, HUEFS, SI); ídem, L.P. Queiroz 14569 (CTES, HUEFS, SI).

2. Borreria brownii (Rusby) Standl., Publ. Field Columbian Mus., Bot. Ser. 7: 333. 1931. Spermacoce brownii Rusby, 
Tabla 1. Cuadro de distribución de las especies por bioma [CER (Cerrado), CP (Campos Rupestres, CAA (Caatingas), MA (Mata Atlántica)]; MAL (malezas), DI (datos insuficientes).

\begin{tabular}{|c|c|c|c|c|c|c|}
\hline Espécies & CER & $\mathrm{CP}$ & CAA & MA & MAL & DI \\
\hline Borreria bahiana & & $\mathrm{X}$ & & & & \\
\hline B. brownii & & & $\mathrm{x}$ & & $\mathrm{X}$ & \\
\hline B. capitata & $\mathrm{X}$ & $\mathrm{X}$ & $\mathrm{x}$ & $\mathrm{X}$ & $\mathrm{X}$ & \\
\hline B. catolensis & & $\mathrm{X}$ & & & & \\
\hline B. crispata & $\mathrm{X}$ & & & & & \\
\hline B. cupularis & $\mathrm{x}$ & & & $\mathrm{X}$ & & \\
\hline B. diamantinae & & $\mathrm{X}$ & & & & \\
\hline B. eryngioides & $\mathrm{x}$ & & $\mathrm{x}$ & $\mathrm{x}$ & $\mathrm{X}$ & \\
\hline B. humifusa & & & & $\mathrm{X}$ & & \\
\hline B. latifolia & $\mathrm{x}$ & $\mathrm{X}$ & $\mathrm{x}$ & $\mathrm{x}$ & $\mathrm{x}$ & \\
\hline B. marticrovettiana & $\mathrm{x}$ & & & & & \\
\hline B. multiflora & $\mathrm{x}$ & & & & & \\
\hline B. ocymifolia & & & $\mathrm{x}$ & $\mathrm{x}$ & $\mathrm{X}$ & \\
\hline B. ocymoides & & & & $\mathrm{X}$ & & \\
\hline B. oligodonta & & & & $\mathrm{X}$ & & \\
\hline B. palustris & & & & $\mathrm{X}$ & & \\
\hline B. paraensis & & & & & & $\mathrm{X}$ \\
\hline B. poaya & $\mathrm{x}$ & & & & & \\
\hline B. prostrata & $\mathrm{x}$ & & & $\mathrm{X}$ & $\mathrm{x}$ & \\
\hline B. pulchristipula & $\mathrm{x}$ & $\mathrm{X}$ & & & & \\
\hline B. reflexa & $\mathrm{x}$ & & & & & \\
\hline B. remota & & $\mathrm{X}$ & & $\mathrm{X}$ & & \\
\hline B. scabiosoides & $\mathrm{X}$ & & $\mathrm{X}$ & $\mathrm{X}$ & & \\
\hline B. schumannii & & & & $\mathrm{X}$ & & \\
\hline B. spinosa & $\mathrm{x}$ & & $\mathrm{X}$ & $\mathrm{X}$ & $\mathrm{x}$ & \\
\hline B. tenella & $\mathrm{x}$ & & & & & \\
\hline B. verticillata & & $\mathrm{X}$ & $\mathrm{x}$ & $\mathrm{x}$ & $\mathrm{x}$ & \\
\hline B. virgata & & & & $\mathrm{X}$ & & \\
\hline B. wunschmannii & $\mathrm{x}$ & & & & & \\
\hline
\end{tabular}

Mem. Torrey Club 4: 208. 1895. Tipo: Bolivia, Yungas, 1890, A.M. Bang 473 (Holotipo NY!; Isotipos MO!, R!).

Fig. 5B-C y 7

Vive desde México hasta el NW de Argentina y Brasil (Bahia, Ceará y Maranhão). En el área de estudio es el primer registro y fue colectada en estepa arbórea abierta dentro del Bioma Caatinga. Es una especie anual comúnmente encontrada como maleza en los cultivos de arroz y de maíz en numerosos países. Florece y fructifica en verano.

Material seleccionado: BRASIL. Bahia: Campo Formoso, 10¹7’S, 40³9’W, 21/IV/1981, W.N. Fonseca 377 (CTES); Camamu, Barcelos do Sul, interior da mata, 18/ VII/2005, A.M. Miranda \& M.I. Silva 5179 (HST, HUEFS); Jaguarari, Cutuni, vale do Morro Redondo, $10^{\circ} 8^{\prime} \mathrm{S}, 40^{\circ} 13^{\prime} \mathrm{W}$, 27/VI/2005, R.F. Souza-Silva \& A. Rapini 45 (HUEFS).
3. Borreria capitata (Ruiz \& Pav.) DC., Prodr. 4: 545. 1830. Spermacoce capitata Ruiz \& Pav., Fl. Peruv. 1: 61, p. 91, fig. B. 1798. Tipo: Perú, 1826, J.A. Pavón s/n (Holotipo MA; Isotipos F!, G!).

Fig. 5D y 7

Habita en Sudamérica, en Bolivia, Brasil, Guayana Francesa, Perú, Venezuela. En Brasil, crece en casi todos los estados y es más frecuente en los biomas del Cerrado s.l., Mata Atlántica (restinga) y Caatinga. Es una especie aparentemente anual y de amplia distribución en Bahia. En este estado crece en Campos Gerais y Campos Rupestres. También ha sido reportada como maleza. Florece y fructifica todo el año.

Material seleccionado: BRASIL. Bahia: 1857, J.S. Blanchet 3760 (BR); 1888, A.F.M. Glaziou 17240 (BR); Piatã, 


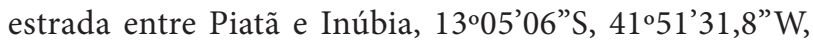
21/V/1999, V.C. Souza et al. 23035 (CTES, ESA); Abaíra, Salão, entre Catolés-Barra, 1200 m, 16/IV/1992, W. Ganev 509 (CTES, HUEFS, K).

4. Borreria catolensis E.L. Cabral \& L.M. Miguel, sp. nov.

Tipo: Brasil, Bahia, Abaíra, distrito de Catolés, encosta

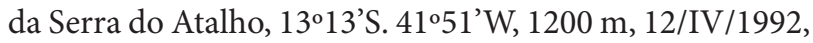
W. Ganev 108 (Holotipo HUEFS!; Isotipos K, SP!).

Borreria catolensis E.L. Cabral \& L.M. Miguel Borreriae gracillimae DC. affinis, sed foliis acicularibus (non linearibus), bracteis $15-25 \mathrm{~mm}$ longis (non 10-15 mm), corolla campanulata (non corolla infundibuliformi basin ventricosa), corollae tubo intus annulum pilosum praedito (tubo intus non piloso), lobis tubum aequantibus (non lobis quam tubo minoribus), staminis faucis adnatis, staminis corollae lobos aequantibus (non staminis tubo suprabasifixis, staminis subsessilis), stylis corollam aequantibus (stylis subsessilis) differt.

Fig. 1A-F y 7

Sufrútice 35-60 cm alt., raíces leñosas, con 5-10 tallos principales que terminan en 1 o 2 ejes floríferos, tallos tetrágonos a subtetrágonos en los nudos basales, $0,5-1 \mathrm{~mm}$ lat., glabros, ángulos violáceos; entrenudos 2-2,3 cm long. Hojas 20-35 x 0,5-1 mm, aciculares, sésiles, glabras, borde revoluto, nervios secundarios inconspicuos; vaina estipular 1-1,2 mm long., glabra, 4 o 5 lacinias de 0,3-3,5 mm long., glabras. Inflorescencias glomeriformes, multifloras, apicales, con 2 o 3 brácteas involucrales de 15-25 mm long., más largas que el glomérulo. Hipanto glabro, ca. 1,6 mm long. Cáliz 4-partido, segmentos 0,8-1 mm long., triangulares, glabros, con dientes en los senos intercalares. Corola blanca, campanuliforme, 4-lobada, 4-4,5 mm long., lóbulos 2-2,5 $\mathrm{mm}$ long., iguales o más cortos que el tubo, con anillo de pelos moniliformes en el interior del tubo corolino cerca de la base y papilas en el tercio superior sobre el dorso de los lóbulos; estambres ca. 2,4 mm long., del mismo tamaño que los lóbulos de la corola, anteras ca. $1 \mathrm{~mm}$ long., contorno oblongo, filamentos ca. 1,2 mm long.; estilo ca. $4 \mathrm{~mm}$ long., capitado bilobado; disco entero. Fruto glabro, inmaduro.

Paratipos: BRASIL. Bahia: Piatã, arredores de Piatã,

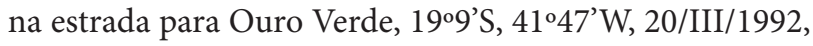
B. Stannard et al. 52727 (K); Piatã, 13/II/1987, R.M. Harley et al. $24162(\mathrm{~K})$.

Borreria catolensis es afín a B. gracillima DC., porque ambas especies son sufrútices con tallos simples o con escasas ramificaciones laterales, de hojas angostas, sin braquiblastos y los glomérulos terminales, raro subterminales. De $B$. gracillima se diferencia porque ésta tiene glomérulos con 2 o 3 brácteas involucrales de 10-15 mm long., corola infundibuliforme basalmente ventricosa, interiormente cubierta con pelos moniliformes, los lóbulos mucho más cortos que el tubo y estambres y estigma inclusos.

Los tres especímenes provienen principalmente de áreas de campos rupestres en el distrito de Catolés, vegetación que en esta región se encuentran bien representada entre 1200-1700 m. Se caracteriza por suelos arenoso-pedregosos, en ocasiones anegadizos, con numerosos afloramientos rocosos. La vegetación predominante está representada por plantas leñosas, de porte pequeño y perennifolias, las hojas, tallos y raíces presentan generalmente adaptaciones xeromórficas (hojas esclerificadas, coriáceas, suculentas o con indumentos particulares), lo que les permite sobrevivir a los grandes cambios térmicos, a la gran variabilidad hídrica y a los efectos del fuego. (Zappi et al. 2003). En este sentido, B. catolensis presenta las adaptaciones necesarias, puesto que es una hierba grácil, con hojas aciculares levemente crasas (sección subcircular) y con raíces leñosas desarrolladas.

Hasta el presente se conoce la distribución restringida a la Serra de Catolés, $1200 \mathrm{~m}$ s.m., vive en campos rupestres o en áreas de transición campos rupestres-cerrados de altitud, en suelo arcilloso o arenoso con afloramientos rocosos. Florece y fructifica de enero a abril.

5. Borreria crispata (K. Schum.) E.L. Cabral \& Bacigalupo, Bonplandia 10: 126. 2000. Borreria tenella (Kunth) Cham. \& Schltdl. var. crispata K. Schum. in Martius, Fl. Bras. 6(6): 55. 1888. Tipo: Brasil, Goiás, s/d, G. Gardner 4175 (Lectotipo P!, desigando por Cabral \& Bacigalupo 2000; Isolectotipos BM!, BR!, NY!).

Fig. 1G-N, 5E-F y 7

Esta especie es endémica de Brasil (Bahia, Goiás, Minas Gerais y Tocantins). En Bahia, se distribuye en la región Centro-Oeste; es una especie propia del Cerrado, preferentemente en campos limpos (veredas de buriti, Mauritia flexuosa L.f.) y campos cerrados. Florece y fructifica de enero a mayo.

Material seleccionado: BRASIL. Bahia: Barreiras, BR$020 \mathrm{em}$ direção a Luis Eduardo Magalhães, a $20 \mathrm{~km}$ de Barreiras, $12^{\circ} 06^{\prime} 41^{\prime \prime}$ ' 45 4509'47”W, 12/IV/2005, E.B. Souza et al. 1147 (CTES, HUEFS); pr. a Cariparé, $11^{\circ} 38^{\prime} \mathrm{S}, 45^{\circ} 15^{\prime} \mathrm{W}$, 5/IV/1978, F.E.L. Miranda 252 (RB); pr. a Serra Geral, 4/ IV/1978, F.E.L. Miranda 237 (RB); ídem, 4/IV/1978, F.E.L. Miranda 232 (RB); $23 \mathrm{~km} \mathrm{~W}$ of Barreiras, 3/III/1972, W.R. Anderson et al. 36557 (UB); Chapadão Ocidental da Bahia, $30 \mathrm{~km} \mathrm{~N}$ de Correntina, 29/IV/1980, R.M. Harley 21944 (UB, UEC); Veredãozinho, río Correntina, 133'소 $45^{\circ} 30^{\text {' }}$ W, 2/II/1967, I.S. Gottsberger 18-2267 (ULM).

6. Borreria cupularis DC., Prodr. 4: 543. 1830. Tipo: Brasil, 1828, J.P. Pohl s/n (Holotipo G-DC!; Isotipo K!).

Fig. 2A-E y 7

Habita en Perú, Bolivia, Paraguay y Brasil (Bahia, Distrito Federal, Goiás, Minas Gerais, São Paulo, Tocantins). En Bahia, la mayor parte de las colecciones proviene de la región Centro-Oeste (Cerrado s.s. o Cerradão), generalmente en contacto con mata ciliar, en suelos arenoso-arcillosos. Sin embargo, se adapta perfectamente a lugares modificados, como borde de caminos o estradas en desuso. El material 
aquí estudiado se menciona como primer registro para Bahia. Florece y fructifica de noviembre a febrero.

Material seleccionado: BRASIL. Bahia: Barreiras, BR-020 em direção a Luis Eduardo Magalhães, a 7 km de Barreiras, $12^{\circ} 06^{\prime} 55^{\prime \prime}$ 'S, $45^{\circ} 04^{\prime} 09^{\prime \prime} \mathrm{W}, 474 \mathrm{~m}$, s/d, E.B. Souza 1133 (CTES, HUEFS).

7. Borreria diamantinae R.M. Salas \& E.L. Cabral, sp. nov. Tipo: Brasil, Abaíra, Campo de Ouro Fino, $13^{\circ} 15^{\prime} \mathrm{S}$, $41^{\circ} 54^{\prime} \mathrm{W}, 1700 \mathrm{~m}$ alt., 21/I/1992, D.J.N. Hind \& R.F. Queiroz 50925 (Holotipo HUEFS!; Isotipos CEPEC, CTES!, K, SPF).

Borreriae humifusae affinis, sed foliis isomorphis (non heteromorphis), glomerulis brevipedunculatis (non glomerulis longipedunculatis), bracteis ellipticis (non bracteis cordatis), seminis transverse sulcatis cum elaiosoma (seminis non transverse sulcatis sine elaiosoma) differt.

Fig. 2F-R y 7

Hierba postrada de tallos radicantes, estoloníferos, extensos y densos con ejes floríferos erectos, entrenudos de 1,5-5,5 cm long., braquiblastos notables en casi todos los nudos. Tallos tetrágonos a subtetrágonos, pelos en los ángulos, más densos en los tallos jóvenes y cerca de la vaina foliar. Hojas 12-30 x 5-15 mm, elípticas o elíptico-ovadas, ápice agudo y base atenuada, discoloras, envés verde-claro, haz pubérula, envés glabro, margen escabriúsculo, 3-6 nervios secundarios curvos hacia el ápice. Vaina estipular 2-3 mm long., pubérula en el margen superior y con pelos densos adpresos en el centro como continuación de las aristas del tallo, con 6-8 lacinias de 2-4 mm long., con notables coléteres apicales. Glomérulos densifloros apicales o subapicales de 5-10 mm lat., con 4-6 brácteas involucrales elípticas o elíptico-ovadas, 2 pares más largas que el glomérulo ( 2 mayores y 2 menores), a veces con 1 par de brácteas triangulares de 3-6 mm long., menores que el glomérulo y ubicada entre las flores, bractéolas filiformes que envuelven parcialmente la base del pedicelo. Hipanto glabro o pubescente solo en el ápice. Cáliz 4-partido, segmentos 1,5-2 mm long., levemente desiguales entre sí, subulados, borde escabriúsculo en la mitad inferior. Corola blanca, infundibuliforme, 4-lobada, 4-5,5 mm long., lóbulos levemente más cortos que el tubo, externamente glabra, con papilas cerca del dorso apical de los lóbulos, internamente con pelos moniliformes en lóbulos y con anillo de pelos moniliformes en el tercio inferior del tubo; estambres y estilo exertos, anteras $1,5 \mathrm{~mm}$ long., contorno oblongo, filamentos 1,2-2 mm long.; estilo 5-6 $\mathrm{mm}$ long., capitado-bilobado; disco 2-partido, cada parte 2-lobulada. Cápsula 3-3,3 mm long., uniseminada, con mericarpos dehiscentes, glabros o pubérulos en el tercio superior. Semilla 1,5-2,5 mm long., castaño oscura, ruminada, retículo-foveada, cara ventral con surco longitudinal angosto cubierto por el estrofíolo que sobresale en la porción inferior, con numerosos paquetes de rafidios y prominente elaiosoma apical cónico.

Paratipos: BRASIL. Bahia: Distrito de Catolés, Mato do Bem Querer, Tanque do Garimpo, $13^{\circ} 16^{\prime} \mathrm{S}, 41^{\circ} 53^{\prime} \mathrm{W}$,
1480 m, 14/V/1992, W. Ganev 267 (HUEFS, K); Lençóis, Barro Branco, Parque Nacional de Chapada Diamantina, $12^{\circ} 20^{\prime} 30^{\prime \prime S}, 41^{\circ} 30^{\prime} 30^{\prime \prime} \mathrm{W}, 5 / \mathrm{IX} / 2000$, R. Filho \& L.C. Soaras 103 (HUEFS).

Esta es la única especie conocida que presenta la combinación de semillas ruminadas con elaisoma, reflejando de cierta manera que la separación realizada por Bacigalupo \& Cabral (1996) entre Borreria ser. Laeves Bacigalupo \& E.L. Cabral y ser. Brachystemonoides Bacigalupo \& E.L. Cabral es artificial (semillas ruminadas vs. no ruminadas con elaiosomas). Además presenta la particularidad de tener una única semilla desarrollada por fruto, la que aparentemente es abortada desde botón, ya que en ese estado uno de los carpelos carece de óvulo.

De la especie más cercana, Borreria humifusa, se diferencia por los caracteres presentes en la clave general. El material Ganev 267 fue identificado por Zappi et al. (2003) como Diodia sarmentosa SW., de la cual es diferenciada por caracteres del hábito (postrado con nudos radicantes vs. escandente), flores (lóbulos corolinos marcadamente pilosos vs. internamente glabros), fruto (con mericarpos dehiscentes vs. indehiscentes) y semillas (1,5-2,5 mm long. ruminadas y con elaiosomas vs. mayores de 3-4 mm long. con surcos poco profundos en la cara ventral).

Endémica de Bahia, restringida a la Chapada Diamantina, forma tapetes en el borde o interior de la mata de altitud, 1480-1700 m. Florece y fructifica casi todo el año.

8. Borreria eryngioides Cham. \& Schltdl., Linnaea 3: 316. 1828. Tipo: Brasil, Brasilia meridionalis, s/d, F. Sellow $s / n$ (Holotipo B †; Lectotipo L!, aquí designado; Isolectotipos HAL!, W!).

Fig. 5G-H y 8

Habita en Argentina, Bolivia, Paraguay, Uruguay y Brasil (Bahia, Distrito Federal, Goiás, Mato Gosso, Mato Grosso do Sul, Paraná, Rio Grande do Sul, São Paulo y Santa Catarina). Crece preferentemente en cerrado, sin embargo se adapta a ambientes secundarios o antropizados, como bordes de caminos o terrenos de cultivos. Pott \& Pott (1994) señalan a esta especie como indicador de suelo descubierto, por efecto de inundación prolongada o por exceso de pastoreo. Florece y fructifica de enero a julio.

Por considerar que el original de Borreria eryngioides ha desaparecido en el herbario B, se elige como lectotipo, el ejemplar del herbario L, por estar completo y en buen estado de conservación.

Material seleccionado: BRASIL. Bahia: Barreiras, near Barreiras airport, ca. $5 \mathrm{~km}$, NW of Barreiras, $650 \mathrm{~m}$, 4/III/1971, H.S. Irwin 31507 (MO, NY); Anguera, Lagoa 5, margem da Lagoa, $12^{\circ} 11^{\prime} \mathrm{S}, 39^{\circ} 9^{\prime} \mathrm{W}, 15 / \mathrm{IX} / 1996, W$. Melo 1735 (K).

9. Borreria humifusa Mart., Flora 24 (2, Beibl.): 68. 1841. Spermacoce martiana Kuntze, Revis. Gen. Pl. 3(3): 123. 1898, nom. illeg (non Spermacoce martiana 
Colla, Herb. Pedem. 3: 146. 1834). Spermacoce casmae Govaerts, World Checkl. Seed Pl. 2(1): 15. 1996 (non Spermacoce humifusa Willd. ex Roem. \& Schult., Syst. Veg. 3: 530. 1818.). Tipo: Brasil, Bahia: In nemoribus at Cruz de Cosme, 1839, Martii 593 [B. Luschnath 88] (Lectotipo BR!, aquí seleccionado; Isolectotipos BM!, F!, G!, MO!, NY!, W!).

Fig. 3A-L, 5K-L y 8

Es endémica de Brasil (Bahia, Paraíba y Pernambuco). Habita en el interior de las florestas estacionales o como palustre en los márgenes de arroyos. Florece y fructifica de mayo a octubre.

Martius (1841) solo se mencionó la localidad donde fue colectado el material, sin embargo no citó ningún espécimen en el cual basó su estudio. Posteriormente, Schumann (1888) redescribió a Borreria humifusa en base a dos especímenes Luschnath 88 y Blanchet 733, diferenciando al primero como el material que examinó Martius como "Marti. Herb. 593". Todos los especímenes de Luschnath 88 depositados en los herbarios BM!, BR!, F!, G!, MO!, NY! y W! tienen en su cartulina la mención Marti. № 593, correspondiente al herbario de Martius. Por lo tanto, si bien no se mencionó ningún material en el protólogo se puede rastrear su localidad y relación con la colección de Luschnath 88. Debido a que existe material en seis herbarios sin mención de un holotipo, se elige la colección de BR como lectotipo, debido a su buen estado de conservación y porque presenta flores y frutos.

Material seleccionado: BRASIL. Bahia: Cruz de Cosme, in umbrosis, VIII/1821, L. Riedel s.n. (BM); Bahia, 1832, J.S. Blanchet 733 (G); ídem, J.S. Blanchet s.n. (G); Amagosa, Serra do Timbó, $13^{\circ} 05^{\prime}$ S, 39³9'W, 750-900 m, 27/I/07, D. Cardoso et al. 1639 (HUEFS); Jiquiriçá, 1352'S, 39²8'W, 29/IX/2005, J. S. Novais 7 (HUEFS).

10. Borreria latifolia (Aubl.) K. Schum. in Martius, Fl. Bras. 6(6): 61, pl. 80. 1888. Spermacoce latifolia Aubl., Hist. Pl. Guiane 1: 55, pl. 19, fig. 1. 1775. Tardavel latifolia (Aubl.) Standl., Contr. U.S. Natl. Herb. 18(3): 122. 1916. Tipo: French Guiana, s/d, J.B.C.F. Aublet s/n (Holotipo $\mathrm{P}$ !; Isotipos BM, MA).

Spermacoce alata Aubl., Hist. Pl. Guiane 1: 60, pl. 22, fig. 7. 1775. Borreria alata (Aubl.) DC., Prodr. 4: 544. 1830. Tipo: French Guiana, Riviere Aroura, s/d, J.B.C.F. Aublet $s / n$ (Lectotipo P-JJR, designado por Delprete, 2010; Isolectotipo BM).

Borreria bartlingiana DC., Prodr. 4: 544. 1830. Spermacoce bartlingiana (DC.) Hemsl., Biol. Cent.-Amer., Bot. 2(7): 58. 1881. Tipo: México, s/d, T.P.X. Haenke $s / n$ (Holotipo G-DC!).

Borreria perrottetii DC., Prodr. 4: 548. 1830. Tipo: French Guiana, s/d, Perrotet in herb. Dunant $s / n$. (Holotipo G-DC!).

Borreria fockeana Miq., Linnaea 18: 299. 1844. Tipo: Surinam, s/d, H.C. Focke $s / n$ (Holtipo U!; Isotipo BR!).

Fig. $5 \mathrm{M}$ y 8
Esta especie habita en Centroamérica y en casi toda Sudamérica, introducida en África, Asia y Australia (Chaw \& Peng 1987; Dessein 2003). En Brasil, vive en casi todo el país y, en Bahia, se la encuentra en todos los biomas, especialmente abundante en cerrado y campos rupestres. Es una especie invasora y persistente en numerosos cultivos y puede sobrevivir a elevadas concentraciones de Glifosato (Lacerda 2003). Florece y fructifica casi todo el año.

Los ejemplares de esta especie, al igual que $B$. poaya y $B$. schumannii, se tornan verde-amarillentos al secarse.

Steyermark (1974) estableció diferencias entre Borreria latifolia y la especie afin $B$. alata, restringiendo a esta última al norte de Sudamérica. Sin embargo, B. latifolia es una especie muy variable en el porte, indumento del tallo, en el tamaño, forma e indumento de las hojas. Por lo tanto, los caracteres mencionados por Steyermark pueden tratarse como variaciones de una misma especie. El análisis del material original de los basónimos de ambas especies (depositados en $\mathrm{P}$ ), nos permite confirmar que B. latifolia y B. alata son conespecíficos.

Material seleccionado: BRASIL. Bahia: Lençóis, Chapada Diamantina, 26/VI/2000, J.G. Jardim 2531 (CEPEC); Morro do Chapéu, 26/X/1995, A. Amorim et al. 1767 (CEPEC, CTES); Tucano, 16/VI/1994, S.C. Sant-Ana et al. 526 (CEPEC, CTES); Una, Reserva Biológica do Mico-Leão, 14/VIII/1996, M.S. Ferrucci et al. 1052 A (CEPEC, CTES).

11. Borreria marticrovettiana E.L. Cabral, Bonplandia 5: 275. 1983. Tipo: Argentina, Misiones, San Ignacio, Teyú Cuaré, 18/XII/1981, E.L. Cabral et al. 212 (Holotipo CTES!; Isotipo SI!).

Fig. $5 \mathrm{~N}$ y 8

Vive en Argentina, Brasil y Paraguay Oriental, en cerrados con campos arenosos. Hasta el momento sólo se ha encontrado para Bahia una colecta en cerrado modificado. Florece y fructifica de noviembre a enero.

Material seleccionado: BRASIL. Bahia: río Piau, ca. 225 $\mathrm{km}$ SW of Barreiras on road to Posse, $850 \mathrm{~m}, 12 / \mathrm{IV} / 1966$, H.S. Irwin et al. 14614 (CTES, MO).

12. Borreria multiflora (DC.) Bacigalupo \& E.L. Cabral, Opera Bot. Belg. 7: 307. 1996. Diodia multiflora DC., Prodr. 3: 564. 1830. Tipo: Brasil (localidad, fecha, colector y número desconocido) (Holotipo G-DC!).

Fig. 5O-P y 8

Crece en Sudamérica, en Venezuela, Guayana, Colombia, Bolivia, Paraguay y Brasil (Bahia, Distrito Federal, Goiás, Mato Grosso, Mato Grosso do Sul, Minas Gerais, S. Paulo). Constituye el primer registro para el estado de Bahia, habita en Cerrado, en campos limpos circundados por áreas anegadas dominadas por Mauritia flexuosa y Mauritiella aculeata (Kunth) Burret ("veredas de buriti"). Florece y fructifica de diciembre a mayo. 
Material seleccionado: BRASIL. Bahia: Formosa do Rio

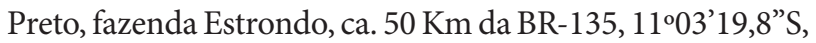
$45^{\circ} 12^{\prime} 0,5^{\prime \prime} \mathrm{W}, 3 / \mathrm{V} / 2009$, L.P. Queiroz et al. 14442 (CTES, HUEFS).

13. Borreria ocymifolia (Willd. ex Roem. \& Schult.) Bacigalupo \& E.L. Cabral, Opera Bot. Belg. 7: 307. 1996. Spermacoce ocymifolia Willd. ex Roem. \& Schult., Syst. Veg. 3: 530. 1818. Diodia ocymifolia (Willd. ex Roem. \& Schult.) Bremek., Recueil Trav. Bot. Neerd. 31: 305. 1934. Hemidiodia ocymifolia (Willd. ex Roem. \& Schult.) K. Schum. in Martius, Fl. Bras. 6(6): 29. 1888. Tipo: West India: "India occidentalis" s/d, Rudolphi $s / n$ (Holotipo B-W!).

Fig. 8

Habita en América Central, Antillas y en América del Sur. En Brasil, vive en casi todo el país y en Bahia principalmente en restinga y en las matas ciliares secundarias. Constituye el primer registro para Bahia. Florece y fructifica de noviembre a marzo.

Material seleccionado: BRASIL. Bahia: Ilhéus, área do CEPEC, km 22 da Rodovia Ilheús-Itabuna (BR 415), 23/II/1981, J.L. Hage \& H.S. Brito 451 (CEPEC, HUEFS); ídem, 28/I/1986, J.L. Hage \& H. S. Brito 1833 (CEPEC, CTES); Pindobaçu, base da Serra da Fumaça, 10³0'42"S, 40²0'31"W, 492 m, 12/IV/2006, R.F. Souza-Silva \& V.J. Santos 148 (HUEFS).

14. Borreria ocymoides (Burm. f.) DC., Prodr. 4: 544. 1830. Spermacoce ocymoides Burm. f., Fl. Ind. 34, pl.13, fig. 1. 1768. Tipo: "Java, Sajor Babi Javanis", s/d, D. Kleynhoff $s / n$ (Holotipo G!).

Borreria repens DC., Prodr. 4: 542. 1830. Spermacoce mauritiana Gideon, Kew Bull. 37:547. 1983 (non Spermacoce repens Willd. ex Cham. \& Schltdl., Linnaea 3: 348. 1828). Tipo: Mauritius, s/d, F.W. Sieber 144 (Holotipo G!; Isotipo P!).

Borreria ocymoides var. bisepala Bremek., Recueil Trav. Bot. Néerl. 31: 307. 1934. Borreria ocymoides fo. bisepala (Bremek.) Steyerm., Acta Bot. Venez. 6: 194. 1971. Tipo: Surinam s/d, J.F Hulk 241 (Holotipo U!).

Borreria exilis L.O. Williams, Phytologia 28(3): 227. 1974. Borreria gracilis L.O. Williams, Phytologia 26(6): 487. 1973, nom. illeg. (non Borreria gracilis Scheele, Linnaea 17: 340. 1843[1844]). Spermacoce exilis (L.O. Williams) C.D. Adams ex W.C. Burger \& C.M. Taylor, Fieldiana, Bot. n.s., 33: 316. 1993. Tipo: Costa Rica, Cocos Island, 28/VI/1932, J.T. Howell 10178 (Holotipo F!).

Fig. $3 \mathrm{M}-\mathrm{U}, 6 \mathrm{~A}$ y 8

Habita en África y Asia Tropical, islas del Pacífico e Índico, en América desde México, Antillas, Surinam, Venezuela hasta Brasil (Amazonas, Bahia, Ceará, Paraíba). Crece aparentemente en el borde o interior de la mata, en lugares muy húmedos. Florece y fructifica casi todo el año.
Borreria ocymoides es una especie pantropical citada en todos los trabajos florísticos de América, África y Asia, en los cuales se involucra a un complejo de especies y nombres, hasta el presente no resuelto. Del análisis del ejemplar tipo y de la descripción original se puede afirmar que su tallo es alado ciliado, con hojas ovadas, pseudoverticiladas, glomérulos axilares y flores con cáliz de 2 sépalos, con estambres y estigma inclusos. Lo observado también coincide con el análisis del tipo de B. repens (Spermacoce mauritiana). Por estos datos, Adams (com. pers. 1995) sostenía que esta especie no estaba representada en América. Sin embargo, la descripción del tipo de B. ocymoides var. bisepala corresponde perfectamente a la var. típica. Por lo tanto, se considera que esta especie también vive en América pero en un área restringida que se extiende desde México, Antillas, Surinam, Venezuela hasta BrasilCon respecto al material americano muy común en los herbarios e identificado como B. ocymoides, se puede afirmar que corresponde a la descripción del tipo de Spermacoce prostrata Aubl. (P). Por lo tanto, Borreria ocymoides se diferencia de B. prostrata por tener tallos glabros alados ciliados (vs. finamente pubescentes), hojas ovadas, pseudopecioladas (vs. elípticas, oblongo-lanceoladas o elíptico-lanceoladas, sésiles), flores con cáliz 2-partido e interior de la corola glabro (vs. cáliz comúnmente 4-partido e interior de la corola con pelos moniliformes en los lóbulos), cápsula glabra o glabrescente (vs. pubescente) y semillas con fovéolas transversalmente alargadas (vs. notables fóveas hexagonales, isodiamétricas).

Material seleccionado: BRASIL. Bahia: Ilhéus. Área do CEPEC, $22 \mathrm{~km}$ da rod. Ilhéus-Itabuna BR-415, região de Mata higrófila sul baiano, 11/VIII/1981, T.S. Santos 3620 (CEPEC, HUEFS).

15. Borreria oligodonta Steyerm., New York Bot. Gard. 23: 826. 1972. Tipo: Venezuela, Bolivar, XII/1938, G.H. Tate 1147 (Holotipo NY!; Isotipo IAN!).

Fig. 8

Crece en Venezuela, Guayanas y Brasil (Bahia y Pará). Es una especie propia de mata de tierra firme de Amazonia de Brasil y Venezuela. En el área de estudio, está aparentemente restringida a las florestas estacionales en el dominio de la mata atlántica. Florece y fructifica de noviembre a enero.

Material seleccionado: BRASIL. Bahia: Caravelas $17 \mathrm{~km}$ de Caravelas para Manuque, campos de restinga, 6/IX/1989, A. Carvalho et al. 2522 (SP); $5 \mathrm{~km}$ na estrada Comandatuba, 4/XII/1991, A. Amorim et al. 522 (CEPEC, CTES); Salvador, 30/I/1974, A. Burkart 30377 (CTES, SI).

16. Borreria palustris (Cham. \& Schltdl.) Bacigalupo \& E.L. Cabral, Hickenia 2: 264. 1998. Diodia palustris Cham. \& Schltdl., Linnaea 3: 347. 1828. Spermacoce palustris (Cham. \& Schltdl.) Delprete, Fl. Il. Catarin. 2: 740. 2005. Tipo: Brasil, Rio Grande do Sul, Morro da Policia, pr. Porto Alegre, 14/III/1949, B. Rambo 40486 (Neotipo LIL!). 
Diodia alata Nees \& Mart., Nova Acta Acad. Caes. Leop. Carol. Wied-Neuwied 12: 12. 1824. Dasycephala alata (Nees \& Mart.) Benth. \& Hook. f. ex B.D. Jacks, Index Kew. 2: 719. 1893. [non Spermacoce alata Aubl., Hist. Pl. Gui. 60. 1775; nec Borreria alata (Aubl.) DC., Prodr. 4: 544. 1830:]. Tipo: Brasil, s/d, M. Wied s/n (Holotipo BR!; Isotipos LD, LE, W).

Borreria gymnocephala DC., Prodr. 4: 549. 1830. Diodia gymnocephala (DC.) K. Schum. in Martius, Fl. Bras. 6(6): 16. 1888. Tipo: Brasil, s/d, J.P. Pohl s/n (Holotipo G-DC!). Fig. 6B y 9

Crece solo en América del Sur, sudeste y sur de Brasil, NE de la Argentina, Perú y Colombia. Habita terrenos bajos, anegadizos, pantanosos. Florece y fructifica casi todo el año.

Material seleccionado: BRASIL. Bahia: Belmonte, 23/ IX/1970, T.S. Santos 1124 (CEPEC).

17. Borreria paraensis E.L. Cabral \& Bacigalupo, Darwiniana 37: 268. 1999. Tipo: Brasil, Pará, Marabá, Alto da Serra, 12/V/1982, R. Secco et al. 117 (Holotipo MG!; Isotipos CTES!, NY!).

Fig. 9

Vive en Brasil (Bahia y Pará). En el área de estudio, los datos de etiqueta son insuficientes para su ubicación geográfica. Florece y fructifica de abril a agosto.

El ejemplar registrado como probable tipo de $B$. tenera DC. en K! (Bahia, Pohl 785, foto en HUEFS!), corresponde a $B$. paraensis, como así también el material citado por Schumann (1888) para ese estado. Por lo expuesto, se excluye por el momento a B. tenera de Bahia (Cabral \& Bacigalupo 2000). Ambas especies se diferencian porque $B$. paraensis tiene glomérulos terminales con 2- 4 brácteas (vs. 3-5 glomérulos axilares por tallo florífero con 4-8 brácteas), frutos sin líneas longitudinales de células taníferas y semillas ruminadas (vs. frutos con líneas longitudinales de células taníferas y semillas no ruminadas), tallos glabros (vs. tallos con pelos adpresos).

Material seleccionado: BRASIL. Bahia: Prope mare ad Cabulla s/d, A.F.M. Blanchet 952 (G); ídem., A.F.M. Blanchet $s / n(\mathrm{G}) ;$ ídem, VII/1834, C.P.F. Martius 601 (BR, G, NY, P); Serra da Erada, s/d, J.P. Pohl 785 (K).

18. Borreria poaya (A. St.-Hil.) DC., Prodr. 4: 459. 1830. Spermacoce poaya A. St.-Hil., Plant. Usuel. Bras. 3: tab. 12. 1824. Tipo: Brasil, São Paulo, A. Saint Hillaire s/n (Holotipo P!).

Fig. 6 C y 9

Vive sólo en Sudamérica, Venezuela, Argentina, Uruguay y en Brasil (Distrito Federal, Goiás, Mato Grosso, Mato Grosso do Sul, Minas Gerais, Paraná, Rio de Janeiro, Santa Catarina, São Paulo, Rio Grande do Sul). Es una especie propia del Cerrado, muy abundante en sur y sudeste del país; sin embargo, en Bahia, las escasas colecciones son del sur en el límite con Minas Gerais. Habita en campos limpos, húmedos, de relieve casi plano en cercanías de veredas de buriti (Mauritia flexuosa). Florece y fructifica de octubre a mayo.

Los ejemplares de esta especie se tornan amarillentos al secarse, al igual que B. latifolia y B. schumannii. La gran variación en la forma, tamaño e indumento foliar, se ve reflejada en la sinonimia de la especie (Cabral \& Salas 2010).

Material seleccionado: BRASIL. Bahia: Cocos, fazenda Trijunção, 16/V/2001, M.L. Fonseca et al. 2750 (CTES, IBGE).

19. Borreria prostrata (Aubl.) Miq., Stirp. Surinam Select.: 177. 1850. Spermacoce prostrata Aubl., Hist. Pl. Guiane 1: 58, pl. 20, fig. 3. 1775. Tipo: French Guiana. Habitat ad ripas fluviorum, Cette plante croît sur le bord des rivieres, s/d, J.B.C.F. Aublet $s / n$ (Holotipo P; Isotipos BM! G-DC!).

Spermacoce gracilis Ruiz \& Pav., Fl. Peruv. 1: 61, pl. 92, fig. a. 1798. Tipo: Perú, Huanuco, Huanuci montium declivibus, s/d, H. Ruiz \& J.A. Pavón s/n. (Holotipo MA).

Borreria asperula DC., Prodr. 4: 543. 1830. Tipo: Brasil, Bahia, s/d, P. Salzmann s/n (Holotipo G-DC!).

Borreria microphylla DC., Prodr. 4: 544. 1830. Tipo: Brasil, s/d, J.P. Pohl s/n (Holotipo G-DC!).

Borreria ramisparsa DC., Prodr. 4: 544. 1830. Tipo: Brasilia, s/d, J.P. Pohl s/n (Holotipo G-DC!).

Borreria tampicana DC., Prodr. 4: 544. 1830. Tipo: "In Sinu Mexicano ad Tampico de Tamaulipas", s/d, J.L. Berlandier $s / n$ (Holotipo G-DC!).

Mitracarpum puberulum Benth., Hook. J. Bot. 3: 238. 1841. Tipo: French Guiana, s/d, R.H. Schomburgk 394 (Holotipo BM!).

Borreria ovalifolia M. Martens \& Galeotti, Bull. Acad. Roy. Sci. Bruxelles 11: 129. 1844. Tipo: Mexico, s/d, H.G. Galeotti 2606 (Holotipo BR!).

Borreria domingensis Griseb., Cat. Pl. Cub.: 141. 1866. Tipo: Cuba, s/d, C. Wright 2770 (Isotipo NY!).

Spermacoce pringlei S. Wats., Proc. Am. Acad. Arts 25: 152. 1890. Tipo: México, Guadalajara, s/d, C.G. Pringle 2464 (Isotipo F!).

Borreria ocymoides f. tenuis Chodat \& Hassl., Bull. Herb. Boiss., sér. 2 4: 183. 1904. Tipo: Paraguay, “Tobaty”, IX, E. Hassler 6438 (Holotipo G!).

Borreia ocymoides var. minima Chodat \& Hassl., Bull. Herb. Boiss., sér. 2 4: 184. 1904. Tipo: Paraguay, "In paludibus pr. Igatimi”, XI, E. Hassler 5546 (Holotipo G!).

Spermacoce cephalophora Rusby, Bull. New York Bot. Gard. 4: 374. 1904. Tipo: Bolivia, s/d, M. Bang 2862 (Isotipo NY!).

Spermacoce ernstii Fosberg \& D. Powell, Smithsonian Contr. Bot. 45: 29. 1980. Tipo: Columbia, Huila above Galilea, s/d, F.R. Fosberg 19632 (Holotipo US!).

Fig. $6 \mathrm{D}$ y 9

Habita en el Sur de Estados Unidos hasta el NE de Argentina. Florece y fructifica a lo largo del año. La identidad de esta especie es discutida bajo B. ocymoides. 
Material seleccionado: BRASIL. Bahia: Salvador, 26/IV/1983, G. Pinto 126 (CEPEC); Barreiras, BR-020 em direção a Luis E. Magalhães, a $7 \mathrm{~km}$ de Barreiras, $12^{\circ} 06^{\prime} 55^{\prime \prime}$ 'S, 4504'09"W, 474 m, 12/IV/2005, E.B. Souza et al. 1128 (HUEFS).

20. Borreria pulchristipula (Bremek.) Bacigalupo \& E.L. Cabral, Bol. Soc. Argent. Bot. 34(3-4): 151. 2000. Diodia pulchristipula Bremek., Recueil Trav. Bot. Néerl. 33: 713. 1936. Tipo: Suriname, Sipaliwini savanna, Camp XI, near the Brazilian border, 10/XII/1935, J. Rombouts 360 (Holotipo U!; Isotipo US!).

Fig. 6E-F y 9

Crece en Guyana, Surinam, Bolivia, Paraguay y Brasil (Bahia, Distrito Federal, Goiás, Mato Grosso, Pará, São Paulo, Tocantins). Habita en terrenos anegadizos, borde de cursos de agua, bosques en galería, en cerrados o campos rupestres. Florece y fructifica casi todo el año. Esta especie es el primer registro para el estado de Bahia.

Material seleccionado. BRASIL. Bahia: Barreiras, Prainha, margem do Rio das Ondas, $12^{\circ} 08^{\prime} 48^{\prime \prime}$, $45^{\circ} 00^{\prime} 55^{\prime \prime} \mathrm{W}$, $456 \mathrm{~m}, 1 / \mathrm{V} / 2009$, D. Cardoso et al. 2627 (CTES, HUEFS); Rio de Contas, estrada para fazenda Marion, 133' $16^{\prime \prime} S$, $41^{\circ} 45^{\prime} 46^{\prime \prime}$ W, 917 m, 7/II/2004, R.M. Harley et al. 54810 (HUEFS); ídem, 2/I/2000, A.M. Giullieti \& R.M. Harley 1626 (HUEFS); Érico Cardoso, Capão, estrada Porteira em direção à Vereda, 13¹6'52"S, 4208'33"W, 1099 m, 5/ VII/2001, H.P. Bautista et al. 3317 (HUEFS); Piatã, estrada Rio de Contas-Livramiento de Brumado, 1336'33"S, $41^{\circ} 48^{\prime} 42^{\prime \prime} \mathrm{W}, 940 \mathrm{~m}, \mathrm{~s} / \mathrm{d}, \mathrm{N}$. Hind et al. 4273 (ALCB, HUEFS, K); Formosa do Rio Preto, $11^{\circ} 03^{\prime} 08^{\prime \prime} \mathrm{S}, 45^{\circ} 11^{\prime} 27^{\prime \prime} \mathrm{W}, 480 \mathrm{~m}$, 29/IV/2000, F. França et al. 3268 (HUEFS).

21. Borreria reflexa J.H. Kirkbr., Acta Amazon. 10(1): 112. 1980. Spermacoce reflexa (Kirkbr.) Govaerts, World Checkl. Seed Pl. 2(1): 18. 1996. Tipo: Brasil, Bahia, 22 km W of Barreiras, Espigão Mestre, 620 m, 2/III/1972, W. Anderson et al. 36482 (Holotipo UB!; Isotipos MO!, F!, NY!, US!).

Fig. 9

Es endémica de Brasil (Bahia, Goiás y Piauí). En Bahia, habita en la región oeste. Es una especie común en el cerrado, preferentemente en campos limpos (con veredas de buriti, Mauritia flexuosa) y campos cerrados, sobre suelo arenoso-rojizo, entre 673-900 m alt. Florece y fructifica de febrero a abril.

Material seleccionado: BRASIL. Bahia: Formosa do Rio Preto, perto do río Riachão, $11^{\circ} 12^{\prime} 41^{\prime \prime} \mathrm{S}, 45^{\circ} 44^{\prime} 38^{\prime \prime} \mathrm{W}$, s/d, W.R.M. Oliveira et al. 492 (CTES, HUEFS); ca. río Piau, $150 \mathrm{~km}$ SW of Barreiras, $850 \mathrm{~m}, 14 / \mathrm{IV} / 1966$, H.S. Irwin et al. 14818 (RB); Espigão Mestre ca. 100 km W of Barreiras, 750 m, 6/III/1972, W. Anderson et al 36698 (F, NY); Barreiras, rod. BR-020, $15 \mathrm{~km} \mathrm{~W}$ de Roda Velha, 10/III/1979, G.

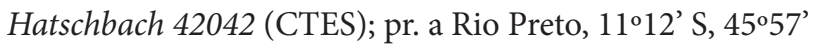

W, 3/III/1978, C. Miranda 213 (RB); São Desidério, 12²8'S, 4512’W, 10/IV/1989, A. Scariot et al. 490 (SP).

22. Borreria remota (Lam.) Bacigalupo \& E.L. Cabral, Darwiniana 37(3-4): 334. 1999. Spermacoce remota Lam., Tabl. Encycl. 1: 273. 1791 [1792]. Tipo: "E Domingo" [República Dominicana], s/d, I. Martín s/n (Holotipo P-LA!).

Fig. 9

Se distribuye en América, desde Estados Unidos, Centroamérica, Antillas hasta Bolivia, Paraguay y Brasil (Bahia, Minas Gerais, Rio de Janeiro, São Paulo). En Bahia, existen escasas colecciones, vive generalmente en el interior o en el borde de florestas y matas. Frecuentemente es hallado como maleza. El material analizado fue encontrado a la sombra de la mata de capão. Florece y fructifica casi todo el año.

Material seleccionado: BRASIL. Bahia: Andaraí, as vertentes das serras ao oeste de Catolés, perto de Catolés

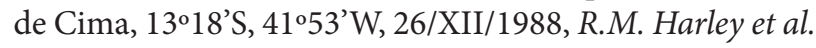
27793 (HUEFS, K).

23. Borreria scabiosoides Cham. \& Schltdl., Linnaea 3: 318. 1828. Spermacoce scabiosoides (Cham. \& Schltdl.) Kuntze, Revis. Gen. Pl. 3(3): 123. 1898. Tipo: Brasil, Rio de Janeiro, F. Sellow s.n. (Holotipo B†, Fot. F 884 en CTES!). Epitipo, aquí seleccionado: Brasil, Bahia, Barreiras, Prainha, margem do Rio das Ondas, $12^{\circ} 08^{\prime} 48^{\prime \prime} S$, 4500'55”'W, 456 m, 1/V/2009, D. Cardoso, R.M. Salas \& A.A. Cabaña-Fader 2626 (HUEFS!, CTES!, K!, SI!).

Fig. 6G-H y 10

Crece en Ecuador, Venezuela, Paraguay, Argentina, Bolivia y Brasil (Amazonas, Bahia, Distrito Federal, Espirito Santo, Pará, Rio de Janeiro, Tocantins). Presenta amplia distribución en Bahia, en casi todos los biomas, Caatinga, Cerrado y Mata Atlántica, en áreas anegadas permanentes o temporales, dentro o al borde de cuerpos de agua lénticos o lóticos. Florece y fructifica a lo largo del año.

El ejemplar tipo citado en el protólogo de Borreria scabiosoides no fue localizado en el herbario (B) y no se hallaron duplicados en otros herbarios europeos consultados. De dicho ejemplar permanece un fototipo (F 884) de buena resolución representado por una rama florífera completa, sin embargo los detalles, de flor y semilla, que caracterizan a esta especie no son observables, razón por la cual se propone un epitipo con numerosos duplicados coleccionado en el estado de Bahia.

Material seleccionado: BRASIL. Bahia: Caetité, ca. 14 $\mathrm{km}$ na estrada de Caetité para Brumado, 19/II/1992, A.M. Carvalho et al. 3769 (CEPEC, CTES); Ilhéus, Praia do Pontal, 30/VII/2001, J.G. Jardim et al. 3723 (CEPEC, CTES); Ilhéus, Campus da Universidade Estadual de Santa Cruz (UESC), $\mathrm{km} 16$ da rod. Ilheús-Itabuna, $14^{\circ} 48^{\prime} \mathrm{S}, 39^{\circ} 10^{\prime} \mathrm{W}, 40$ m, L.A. Mattos-Silva et al. 3147 (HUEFS, UESC); Lençóis, $12^{\circ} 40^{\prime} 03^{\prime \prime S}, 41^{\circ} 19^{\prime} 28^{\prime \prime} \mathrm{W}, 360 \mathrm{~m}, 13 / \mathrm{XII} / 2002$, F. França et al. 3887 (HUEFS). 
24. Borreria schumannii (Standl. ex Bacigalupo) E.L. Cabral \& Sobrado, comb. nov., Diodia schumannii Standl. ex Bacigalupo in Burkart, Fl. Ilustr. Entre Ríos 6(6): 15, fig. 5. 1974. Borreria flavovirens Bacigalupo \& E.L. Cabral, Hickenia 2: 261. 1998, nom. illeg. Spermacoce schumannii (Standl. ex Bacigalupo) Delprete, Fl. Ilustr. Catarin. Rubiaceas 2: 754. 2005. Tipo: Argentina, Entre Rios, La Paz, Isla Curuzú-Chalí, fl. fr., 10/IV/1968, A. Burkart et al. 27103 (Holotipo SI!; Isotipo CTES!).

Fig. 5I-J y 10

En Sudamérica, habita en el NE de Argentina, Paraguay y Brasil ( Bahia, Goiás, Maranhao, Minas Gerais, Paraná, Rio Grande do Sul, Rondonia, Santa Catarina, São Paulo, Tocantins). Crece preferentemente al borde o interior de matas húmedas o ciliares, terrenos ribereños. En Bahia, se encontró en áreas de restinga. Los ejemplares de esta especie, al igual que $B$. poaya y $B$. latifolia se tornan verdeamarillentos al secarse. Florece y fructifica todo el año.

Delprete \& Kirkbride (2008) demostraron que Diodia schumannii es un nombre válidamente publicado. Sin embargo, consideran esta especie bajo Spermacoce schumannii, posición que en este trabajo se discute. Por lo tanto, se realiza la nueva combinación y se traslada el epíteto a Borreria.

Material seleccionado: BRASIL. Bahia: Between Alcobaça and Prado, on the coast road $12 \mathrm{~km} \mathrm{~N}$ of Alcobaça, mixed restinga with high forest, $17^{\circ} 29^{\prime} \mathrm{S}, 39^{\circ} 13^{\prime} \mathrm{W}$, nivel del mar, 16/I/1977, R. M. Harley 17995 (HUEFS, K).

25. Borreria spinosa (L.) Cham. \& Schltdl., Linnaea 3: 340. 1828. Spermacoce spinosa L., Syst. Nat. (ed. 10) 2: 890 1762. [non Spermacoce spinosa Jacq., Select. Stirp. Amer. Hist. 21: 7. 1763, nec Borreria spinosa (Jacq.) DC., Prodr. 4: 542. 1830]. Tipo: Jamaica, s/d, coleccionista desconocido (Holotipo S-LINN!).

Borreria densiflora DC., Prodr. 4: 542. 1830.. Spermacoce densiflora (DC.) Alain, Phytologia 54(2): 113. 1983. Tipo: Jamaica, s/d, O. Swartz s/n (Holotipo G-DC!; Isotipos B!).

Borreria radicosa Brandgee, Univ. Calif. Publ. Bot. 10: 416. 1924. Tipo: México, Chiapas, Jalisco, VII/1923, C.A. Purpus 9223 (Holotipo MO; Isotipo US!)

Borreria densiflora var. perennis Standl., Publ. Field Mus. Nat. Hist., Bot. Ser. 7: 334. 1931. Tipo: Bolivia, Villamontes, 1923, K. Pflanz 2077 (Holotipo US!). Syn. nov.

Borreria densiflora var. pilosa Bacigalupo, A.L. Cabrera, Fl. Prov. Jujuy 9: 413. 1993. Tipo: Argentina, Jujuy, Capital, camino al dique La Ciénaga, s/d, A. Burkart \& N. Troncoso $s / n$ (Lectotipo SI 11086!, aquí seleccionado).

Spermacoce spinosa L. fue descrita por Linneo (1762), aparentemente a partir de un material depositado en el herbario Linneano S (microficha $\mathrm{n}^{\circ}$ : IDC 53.15), en uno de los fototipos disponibles se puede observar que fue colectado en Jamaica. Este material consta de una rama florífera, con cuatro glomérulos, cada uno con numerosas brácteas con papilas romas evidentes sobre el envés en el nervio principal. Si bien el material coincide plenamente con la descripción de S. spinosa, fue determinado como Spermacoce verticillata, identificación asentada en la cartulina y en la base de datos del herbario S-LINN (http://innaeus/ nrm.se/botany/fbo/s/sperm/welcome.html.en). Dos años antes se publicó un homónimo, Spermacoce spinosa Jacq., sin embargo se lo considera nomen nudum debido a que carece de descripción y de una mención de material tipo. El mismo autor en 1763 revalidó la descripción y citó un material proveniente de La Habana, Cuba. Por lo tanto, el binomio propuesto por Linneo es prioritariamente válido. Chamisso \& Schlechtendal (1828), transfirieron el epíteto de Linneo a Borreria como B. spinosa (L.) Cham. \& Schltdl. citando un ejemplar de Jamaica (Swartz $s / n$, isotipo de $B$. densiflora) que se encuentra depositado en B (B-W 2643), nombre aquí considerado como válido.

Candolle (1830) describió a Borreria densiflora en base a un duplicado de Swartz $s / n$ depositado en G-DC , nombre que en este trabajo se lo considera como sinónimo.

Fawcet \& Rendle (1936) fueron aparentemente los primeros en considerar a $B$. densiflora bajo la sinonimia de $B$. spinosa, concepto seguido por Liogier (1962). Éste último autor (Liogier 1997) modificó su opinión y trató a esta especie como Spermacoce densiflora, siguiendo claramente a Steyermark (1972). Steyermark propone que S. spinosa de Linneo es un nombre ambiguo, porque en la descripción cita como sinónimos a S. suffruticosum Loefl. (nomen nudum, sin mención de tipo) y a S. spinosa Jacq. y además por la dificultad que tuvo en encontrar el ejemplar tipo de Linneo. Señala también que el único material identificado como S. spinosa, microficha $\mathrm{n}^{\circ}$ : IDC 125.1 , se corresponde a $S$. tenuior L. (sin embargo, en S-LINN está identificado como Thapsia trifoliata L., Apiaceae). Por esta razón, Steyermark (1972) concluyó que B. densiflora DC. es el nombre válido y más antiguo para esta especie. El argumento de Steyermark se basaba en la inexistencia de un material tipo en S-LINN; sin embargo, en este trabajose confirma que existe un holotipo en S-LINN. Por lo tanto, se considera como combinación válida para esta especie a B. spinosa (L.) Cham. \& Schltdl.

Clave para identificar las variedades de Borreria spinosa

1. Tallo simple o con ramas distales; hojas $0,2-1(-1,5)$ $\mathrm{cm}$ lat., linear, linear-lanceolada, linear-oblonga o linear-elíptica, sésiles, nervios secundarios inconspicuos 25a. B. spinosa var. spinosa

1. Tallo con numerosas ramas opuestas desde la base. Hojas de 0,8-2,5 cm lat., elípticas o lanceoladas con base atenuada en pseudopecíolo, 3 nervios secundarios visibles en el envés. 25b.B. spinosa var. latifolia

\section{5a. Borreria spinosa (L.) Cham. \& Schltdl. var. spinosa.} Fig. 6I-J y 10.

Se distribuye en América, desde México, Antillas hasta el centro de Argentina. En Bahia, es una especie ruderal, común en ambientes secundarios como bordes de camino o 
campos labrados, se desarrolla bien en Cerrado o Caatinga. Florece y fructifica casi todo el año.

Material seleccionado: BRASIL. Bahia: Casa Nova, fazenda Santarém, sitio Morrinho, área vazante do lago

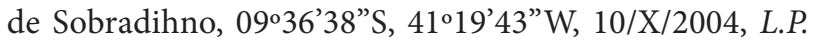
Queiroz 9664 (CTES, HUEFS).

25b. Borreria spinosa (L.) Cham. \& Schltdl. var. latifolia (E.L. Cabral \& Martins) E.L. Cabral, comb. nov. Borreria densiflora var. latifolia E.L. Cabral \& Martins, Weed Biol. Manag. 9: 286. 2009. Tipo: Brasil, Tocantins, 08 58'03”S, $48^{\circ} 10^{\prime} 29^{\prime \prime}$ W, 8/V/2007, V.C. Souza \& B.A.B. Martins 32954 (Holotipo ESA!; Isotipo CTES!).

Vive en el centro este y nordeste de Brasil (Bahia, Ceará, Maranhão, Piauí, Tocantins y Goiás). Con frecuencia, se halla como maleza en cultivos diversos, principalmente en los de soja. Florece y fructifica casi todo el año.

Material seleccionado: BRASIL. Bahia: Lençóis, $12^{\circ} 34^{\prime} \mathrm{S}$, $41^{\circ} 23^{\prime} \mathrm{W}, 450 \mathrm{~m}, 8 / \mathrm{IX} / 2005$, E.B. Souza et al. 1406 (HUEFS); Jaguarari, caminho do Engenho, estrada para Grotas, $10^{\circ} 08^{\prime} \mathrm{S}$, $40^{\circ} 13^{\prime} \mathrm{W}, 24 / \mathrm{VII} / 2005$, R.F. Souza-Silva \& A. Rapini 22 (HUEFS).

26. Borreria tenella (Kunth) Cham. \& Schltdl., Linnaea 3: 317. 1828. Spermacoce tenella Kunth, Nov. Gen. Sp. 3: 345. 1819. Borreria tenella var. tenella (Kunth) K. Schum., Fl. Bras. 6 (6): 55. 1888. Spermacoce suaveolens (G. Mey.) Kuntze var. tenella (Kunth) Kuntze., Revis. Gen. Pl. 3(3): 124. 1898. Tardavel tenella (Kunth) Standl., Contr. U. S. Natl Herb. 18(3): 122. 1916. Borreria capitata var. tenella (Kunth) Steyerm., Mem. New York Bot. Gard. 23: 823. 1972. Tipo: Venezuela. Orinoco, s/d, A. Humbolt \& M.A. Bonpland s/n (Holotipo B-W 2637!) Borreria linoides DC., Podr. 4: 548. 1830. Borreria tenella var. linoides (DC.) K. Schum. in Martius, Fl. Bras. 6(6): 55. 1888. Borreria suaveolens var. linoides (DC.) Standl., Field Mus. Nat. Hist., Bot. Ser. 11(5): 185. 1936. Tipo: Brasil, 1828, J.P. Pohl s/n (Holotipo G-DC!).

Spermacoce aturensis Kunth, Nov. Gen. Sp. (quarto ed.) 3: 345. 1818 [1819]. Borreria aturensis (Kunth) DC., Prodr. 4: 545. 1830. Tipo: Venezuela, río Orinoco, crescit ad fluvium Orinoci, prope Atures, A. Humbolt \& M.A. Bonpland $s / n$ (Holotipo P!).

Spermacoce oronocensis Willd. ex Roem. \& Schult., Syst. Veg., ed. 15 bis 3: 531.1818 [1819]. Tipo: Venezuela, río Orinoco, s/d., A. Humbolt \& M.A. Bonpland $s / n$ (Holotipo B-W!).

Fig. 6K-L y 10

Se distribuye en América, desde Centroamérica hasta el norte de Argentina. En Brasil, es una especie propia del cerrado, muy abundante en los estados de las regiones del Sur, Sudeste y Centro-Oeste del país. En Bahia, existen abundantes colecciones de la región oeste del río São Francisco. Florece y fructifica de octubre a junio.

Originalmente, el ejemplar A. Humbolt \& M.A. Bonpland $s / n$ (B-W 2637) fue identificado como Spermacoce capitata Ruiz \& Pav., que posteriormente Schumann (1888) reconoce como material tipo de Spermacoce tenella. Steyermark (1972), basado en el examen del fototipo, incluye a $B$. tenella como una variedad de B. capitata, concepto seguido en varias floras americanas (Steyermark 1974; Brako \& Zarucchi 1993; Taylor \& Steyermark 2004; Funk et al. 2007). Steyermark (1972) discute la decisión de Schumann de reconocerla como especie aparte y menciona que las diferencias en las semillas (no ruminadas vs. ruminadas) no son evidentes en el material tipo que solo presenta flores. Basado en el análisis del material tipo, se optó por el concepto de especie de Schumann. Este taxón se caracteriza por tener tallos floríferos con indumento variado, simples con escasos brotes axilares, con 1 (raro 2 o 3 ) glomérulos apicales y semillas cilíndricas sin surcos. En este concepto de especie, se reconoce además que a largo de su distribución, $B$. tenella presenta una variación en el tamaño, forma e indumento foliar. Por otro lado, se describe a B. capitata como un sufrútice pubescente, generalmente muy ramificado, con hojas elípticas u ovadas, plegado-nervosas, pseudoverticiladas y las semillas ruminadas.

Material seleccionado: BRASIL. Bahia: $5 \mathrm{~km}$ do limite GO-BA, 135'31"S 4611'22”'W, 993 m, 14/IV/2005, E.B. Souza 1166 (CTES, HUEFS).

27. Borreria verticillata (L.) G. Mey., Prim., Fl. Esseq.: 83. 1818. Spermacoce verticillata L., Sp. Pl. 1: 102. 1753. Tipo: "Spermacoce verticillis globosis", in Dillenius, Hort. Eltham. 2: 369, t. 277, f. 358. 1732 (Lectotipo BM 000557767!, desigando por Rendle 1934).

Fig. $6 \mathrm{M}$ y 10

Habita en América, EE.UU hasta norte de Argentina, introducida en W de África. Es una especie heliófita de amplia distribución en Brasil y crece generalmente al borde de camino, con suelos arenosos en ocasiones muy erosionados, en ambientes secundarios de variadas formaciones vegetales. Florece y fructifica de octubre a mayo.

Borreria verticillata es de hábito muy variable como así también la forma y tamaño de las hojas. Con respecto al fruto, el material analizado del cono sur (Argentina, Uruguay, Paraguay y sur de Brasil) presenta frutos con mericarpos indehiscentes, mientras que el material del norte tiene mericarpo dehiscentes como el material tipo.

Material estudiado: BRASIL. Bahia: 1830, P. Salzman $s / n$. (G-DC); Andaraí, 7 km SW de Nova Redenção, caminho a Mucugê, $12^{\circ} 46^{\prime} \mathrm{S}, 41^{\circ} 11^{\prime} \mathrm{W}, 400 \mathrm{~m}, 25 / \mathrm{XI} / 1992$, M.M. Arbo et al. 5778 (SPF, CTES); Pindobaçu, margen do Rio da Fumaça, 10³9'42”S, 40²0’50”W, 492 m, 12/IV/2006, V.J. Santos 507 (CTES, HUEFS); Itabuna, $10 \mathrm{~km} \mathrm{~S} \mathrm{de} \mathrm{Pontal}$ (Ilhéus), caminho a Olivença, $14^{\circ} 54^{\prime} \mathrm{S}, 39^{\circ} 02^{\prime} \mathrm{W}, 50 \mathrm{~m}$, s/d, M.M. Arbo et al. 5548 (CTES, SPF).

28. Borreria virgata Cham. \& Schltdl., Linnaea 3: 324. 1828. Spermacoce virgata (Cham. \& Schltdl.) Hemsl., Biol. Cent.-Amer., Bot. 2(7): 61. 1881, nom. illeg. (non 

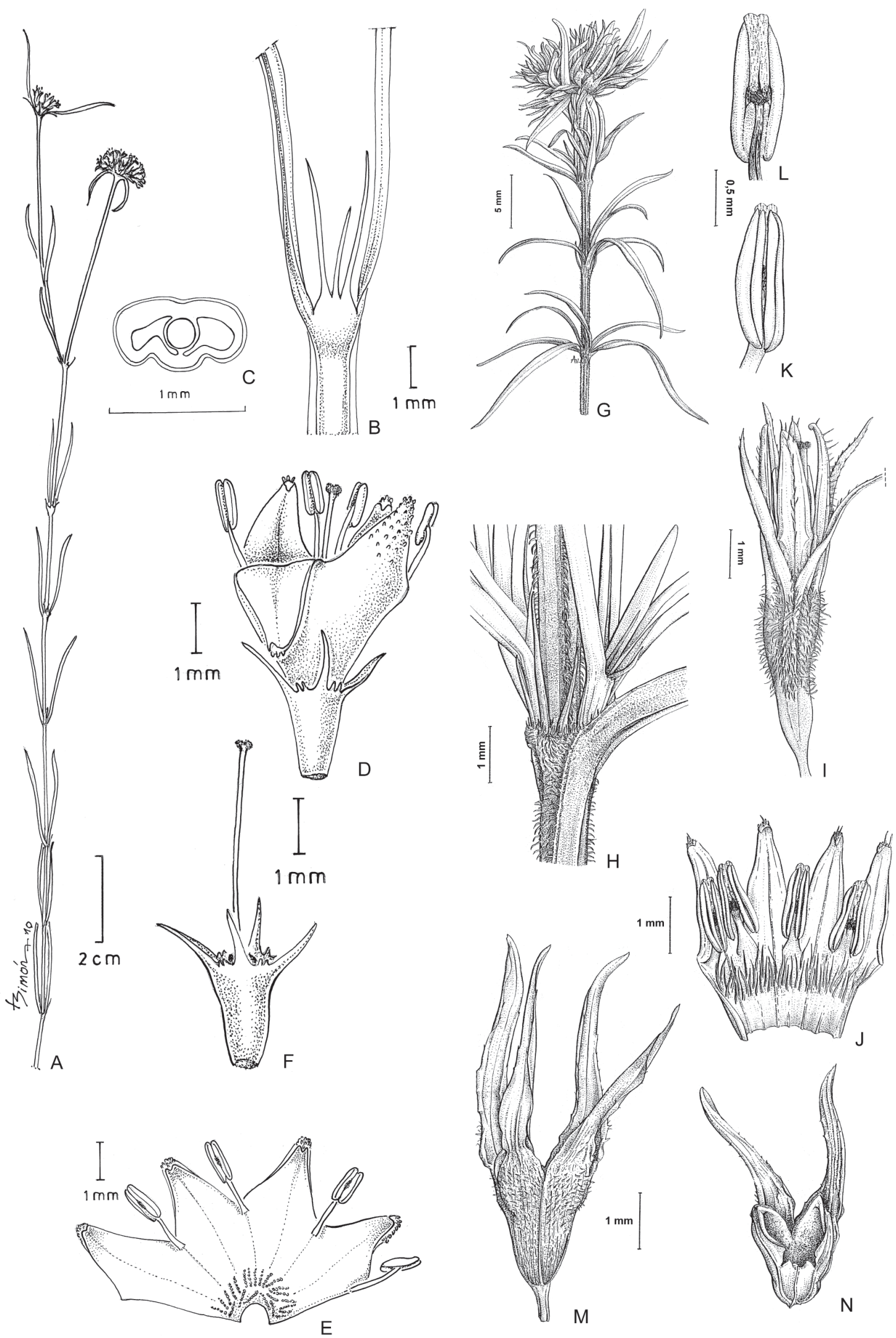

Figura. 1. A-F. Borreria catolensis E.L.Cabral \& L.M. Miguel. A. Hábito. B. Vaina estipular. C. Corte transversal de hoja. D. Flor. E. Corola abierta. F. Hipanto, estilo y estigma. (Ganev 108 SP). G-N. Borreria crispata (K. Schum.) E.L. Cabral \& Bacigalupo. G. Parte de una rama florífera. H. Vaina estipular. I. Flor. J. Corola abierta. K-L. Anteras: K. Cara ventral; L. Cara dorsal con glándula en conectivo. M. Fruto. N. Vista interna de mericarpo (Gardner 4175 BR). 


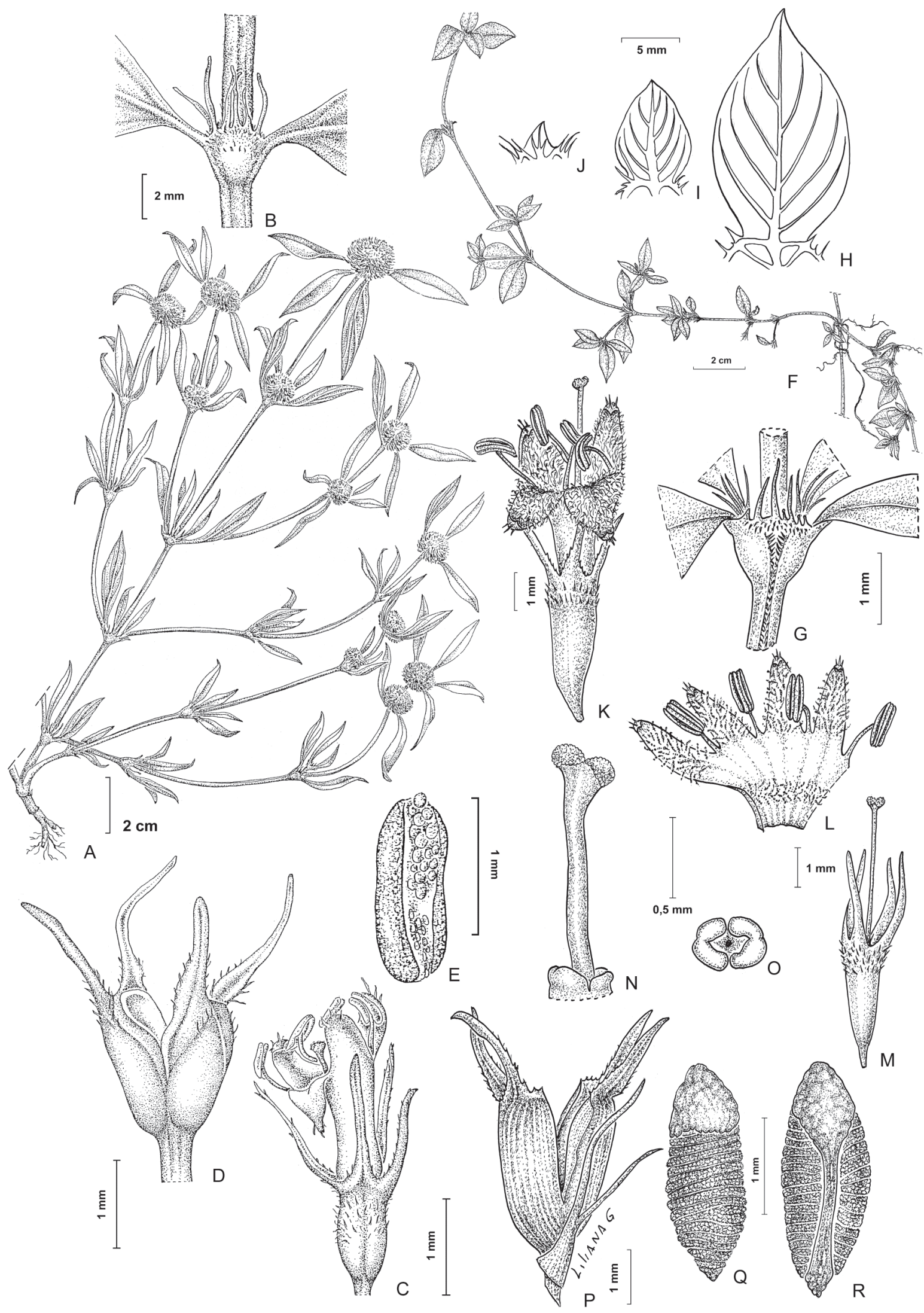

Figura 2. A-E. Borreria cupularis DC. A. Hábito. B. Vaina estipular. C. Flor. D. Fruto. E. Semilla, cara ventral con elaiosoma. (Arbo 3307 CTES). F-R. Borreria diamantinae R.M. Salas \& E.L. Cabral. F. Hábito. G. Vaina estipular. H-J. Brácteas de un glomérulo. K. Flor. L. Corola abierta. M. Hipanto, estilo y estigma. N. Disco, estilo y estigma. O. Disco. P. Fruto. Q-R. Semilla, con elaiosoma: Q. Cara dorsal; R. Cara ventral. (Hind 50925 HUEFS). 


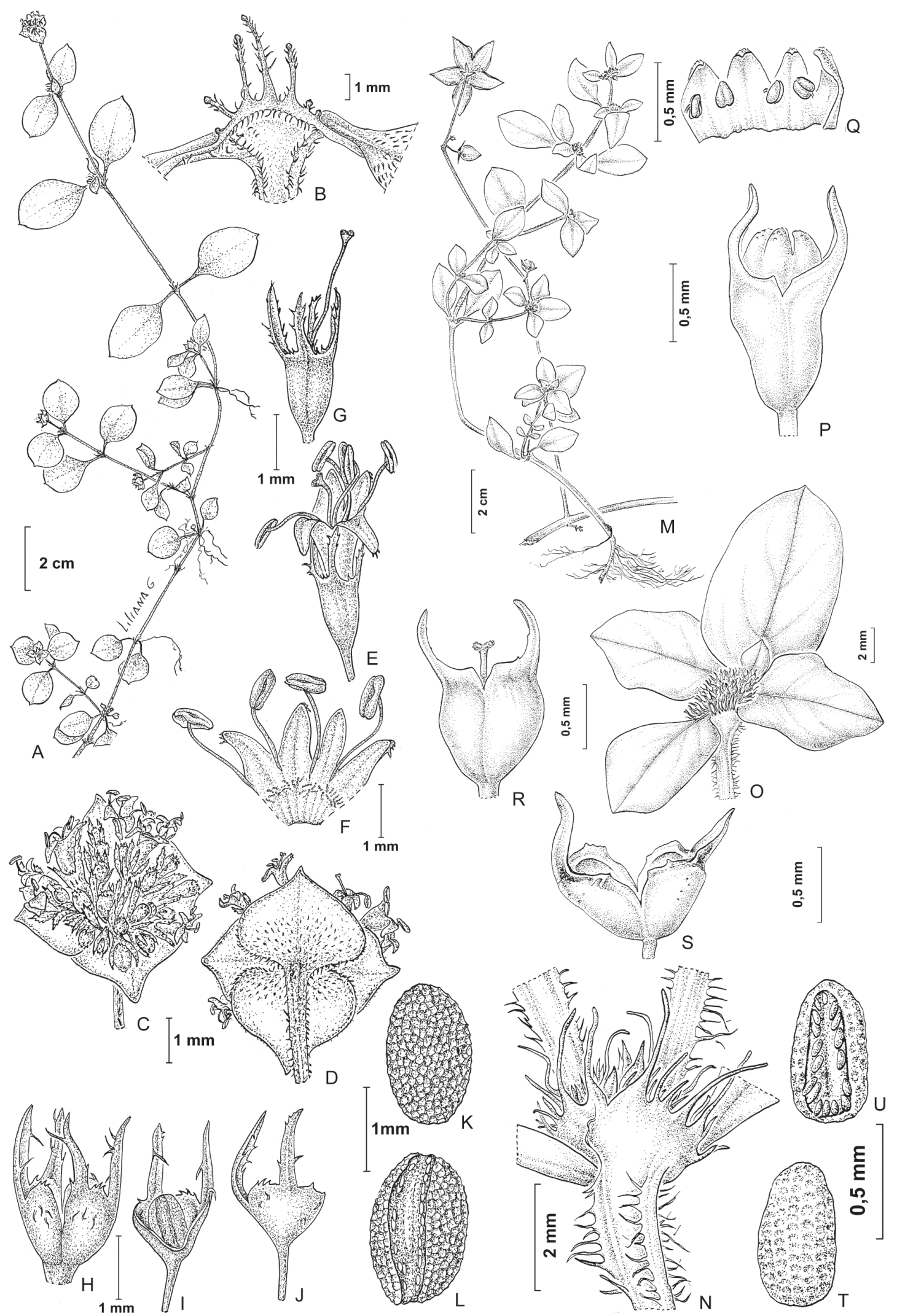

Figura 3. A-L. Borreria humifusa Mart. A. Hábito. B. Vaina estipular. C-D. Glomérulo con brácteas: C. Vista superior; D. Vista inferior. E. Flor. F. Corola abierta. G.Hipanto, estilo y estigma. H. Fruto. I-J. Mericarpo: I. Vista ventral; J. Vista dorsal. K-L. Semilla: K. Cara dorsal; L. Cara ventral. (Sales de Melo 230 CTES). M-U. Borreria ocymoides (Burm. f.) DC. M. Hábito. N. Vaina estipular. O. Glomérulo con brácteas. P. Flor. Q. Corola abierta. R. Hipanto, estilo y estigma. S. Fruto. T-U. Semilla: T. Cara dorsal; U. Cara ventral. (Allemão 806 R; publicado en Cabral \& Bacigalupo 1996). 

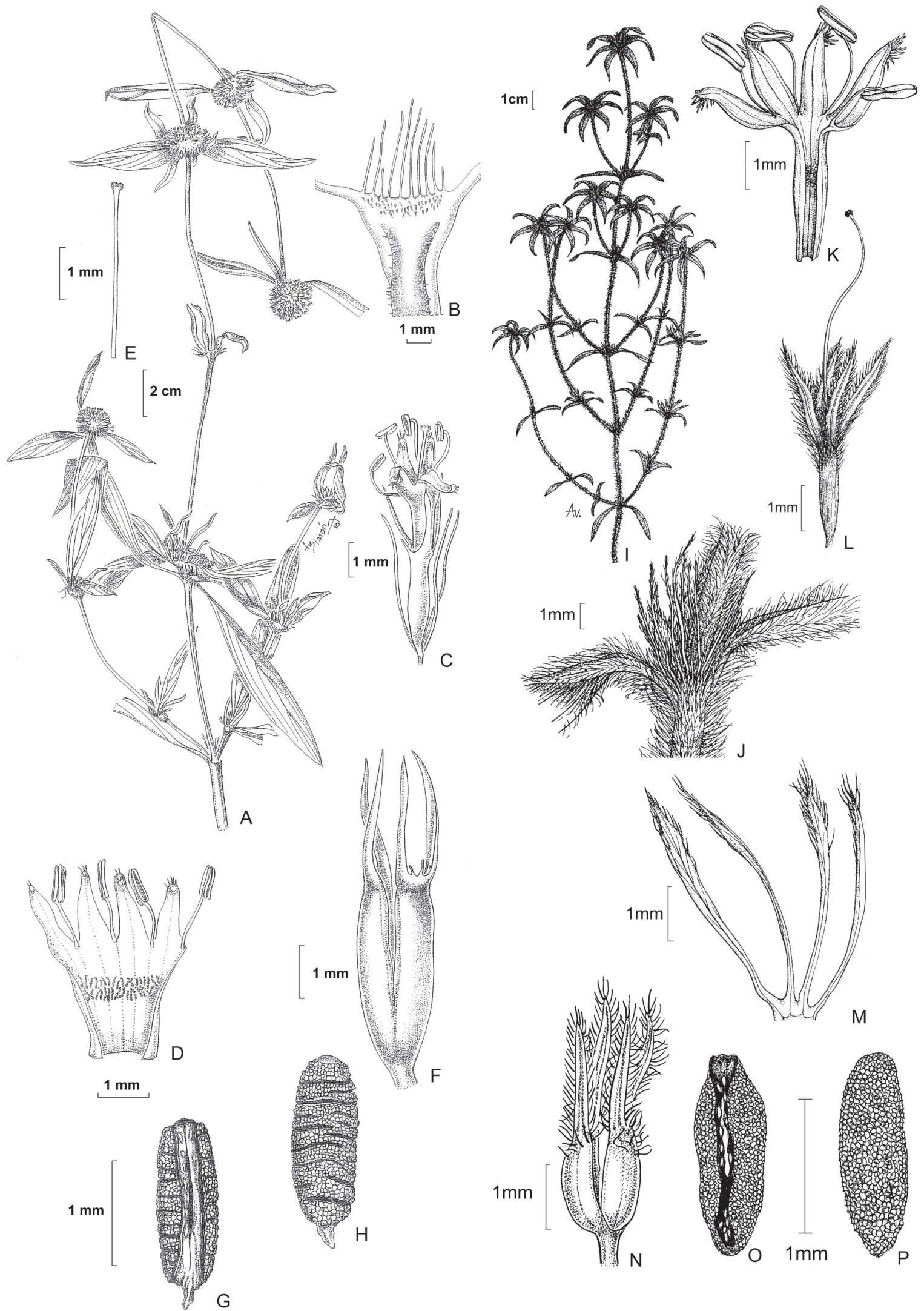

Figura 4. A-H. Borreria virgata Cham. \& Schltdl. A. Rama florífera. B. Vaina estipular. C. Flor con bracteolas. D. Corola abierta. E. Estilo y estigma. F. Fruto. G-H. Semilla: G. Cara ventral; H. Cara dorsal. (Martii 599 MO). I-P. Borreria wunschmannii K. Schum. I. Rama florífera. J. Vaina estipular. K. Corola abierta. L. Hipanto, estilo y estigma. M. Bracteolas. N. Fruto. O-P. Semilla: O. Cara ventral; P. Cara dorsal. (Jardim 146 CEPEC). 

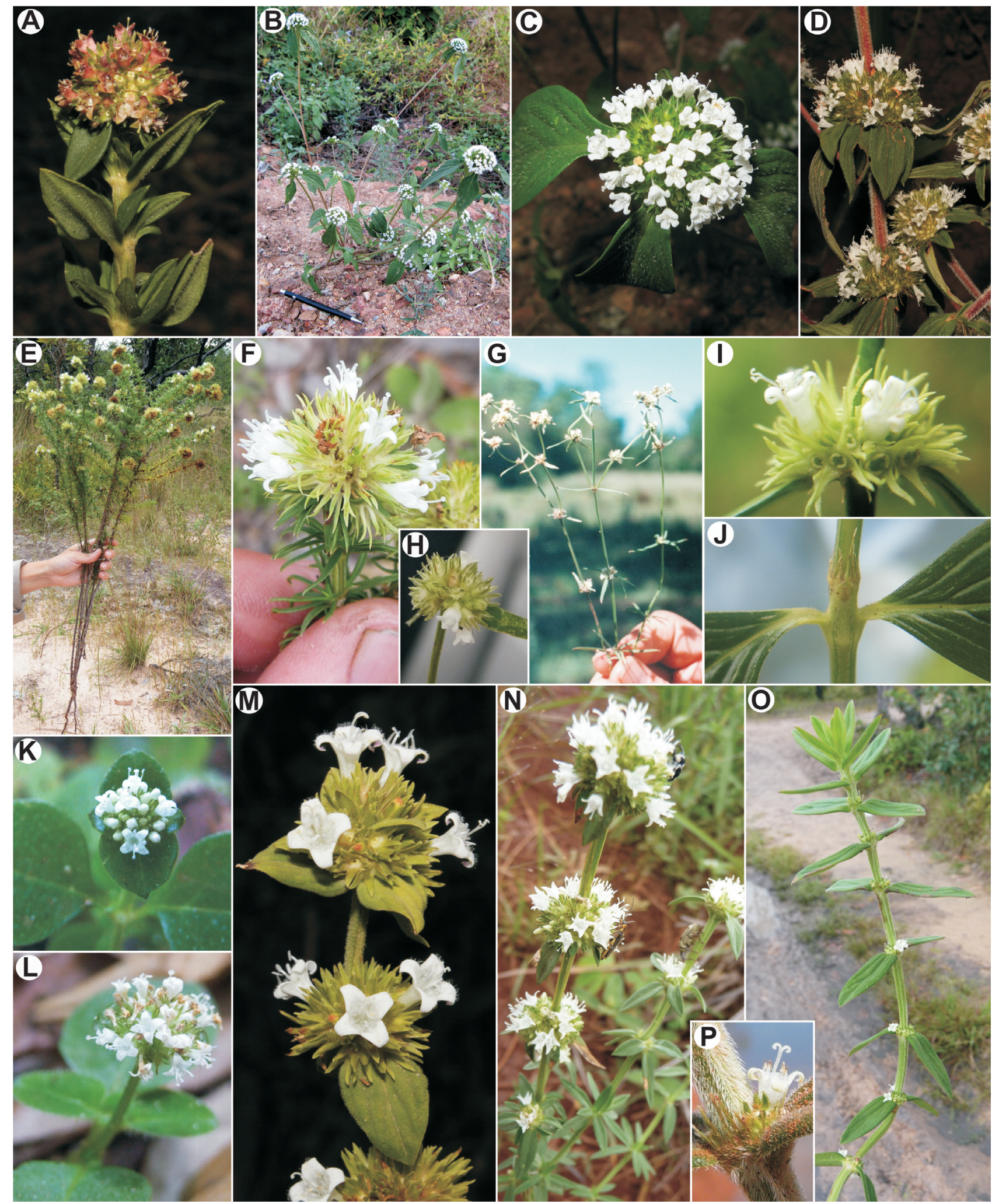

Figura 5. Fotografías de especies de Borreria. A. B. bahiana E.L. Cabral: inflorescencia apical. B-C. B. brownii (Rusby) Standl.: B. Hábito. C. Inflorescencia apical. D. B. capitata (Ruiz \& Pav,) DC.: inflorescencias apical y axilar. E-F. B. crispata (K. Schum.) E.L. Cabral \& Bacigalupo: E. Planta entera. F. Inflorescencia apical. G-H. B. eryngioides Cham. \& Schltdl.: G. Rama florífera. H. Inflorescencia apical. I-J. B. schumannii (Standl. ex Bacigalupo) E.L. Cabral \& Sobrado: I. Inflorescencia axilar. J. Vaina estipular. K-L. B. humifusa Mart.: K. Brácteas cordadas. L. Inflorescencia pedunculada . M. B. latifolia (Aubl.) K. Schum.: inflorescencias apical y axilar. N. B. marticrovettiana E.L. Cabral: inflorescencias apical y axilares. O-P. B. multiflora (DC.) Bacigalupo \& E.L. Cabral: O. Rama florífera. P. Flor con estigma bífido. Créditos fotográficos. A, D, K-M: D. Cardoso; B-C: E.B. Souza; E-F, H -J, N-P: R.M. Salas; G: E.L. Cabral. 

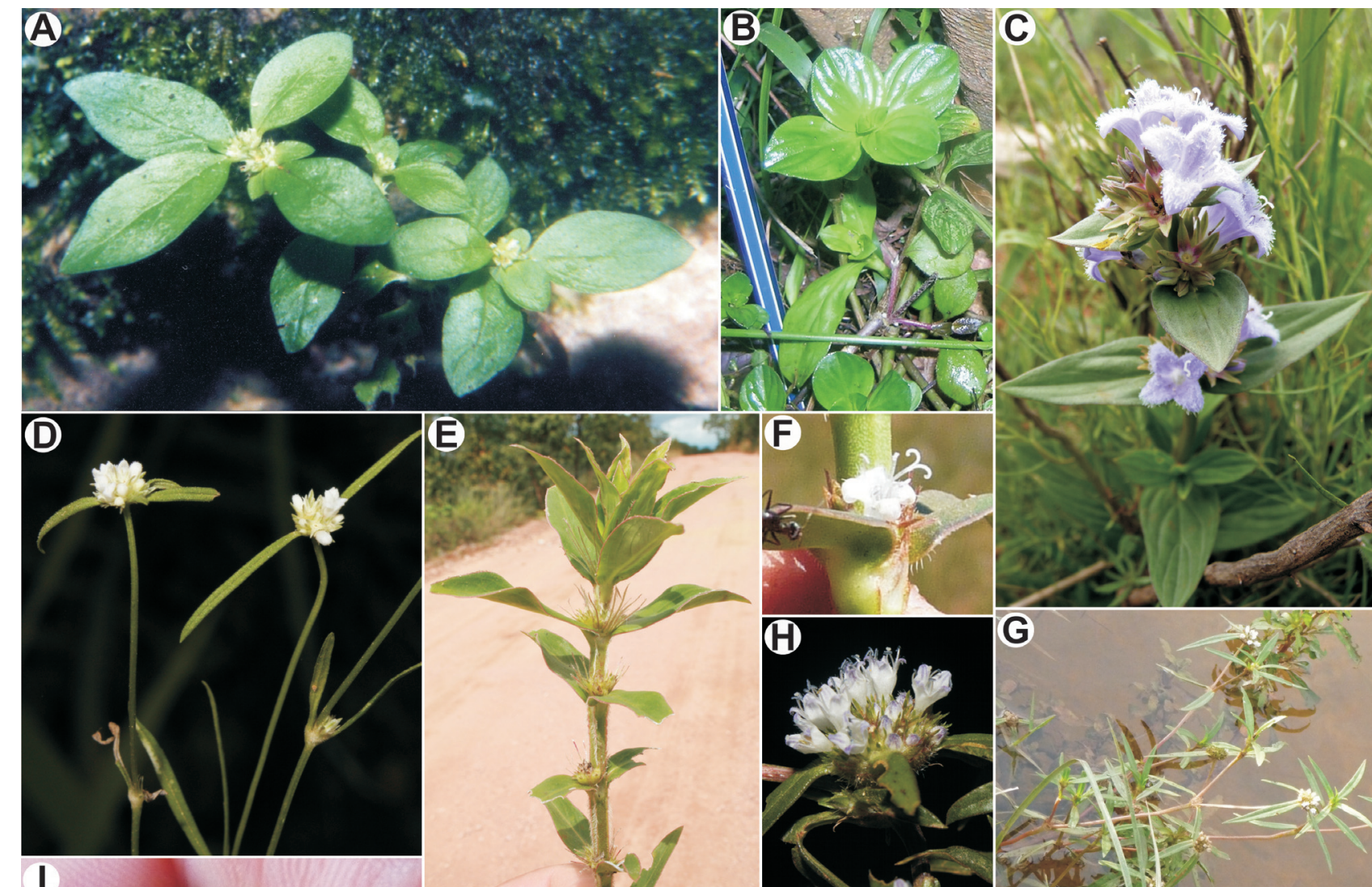

(1)
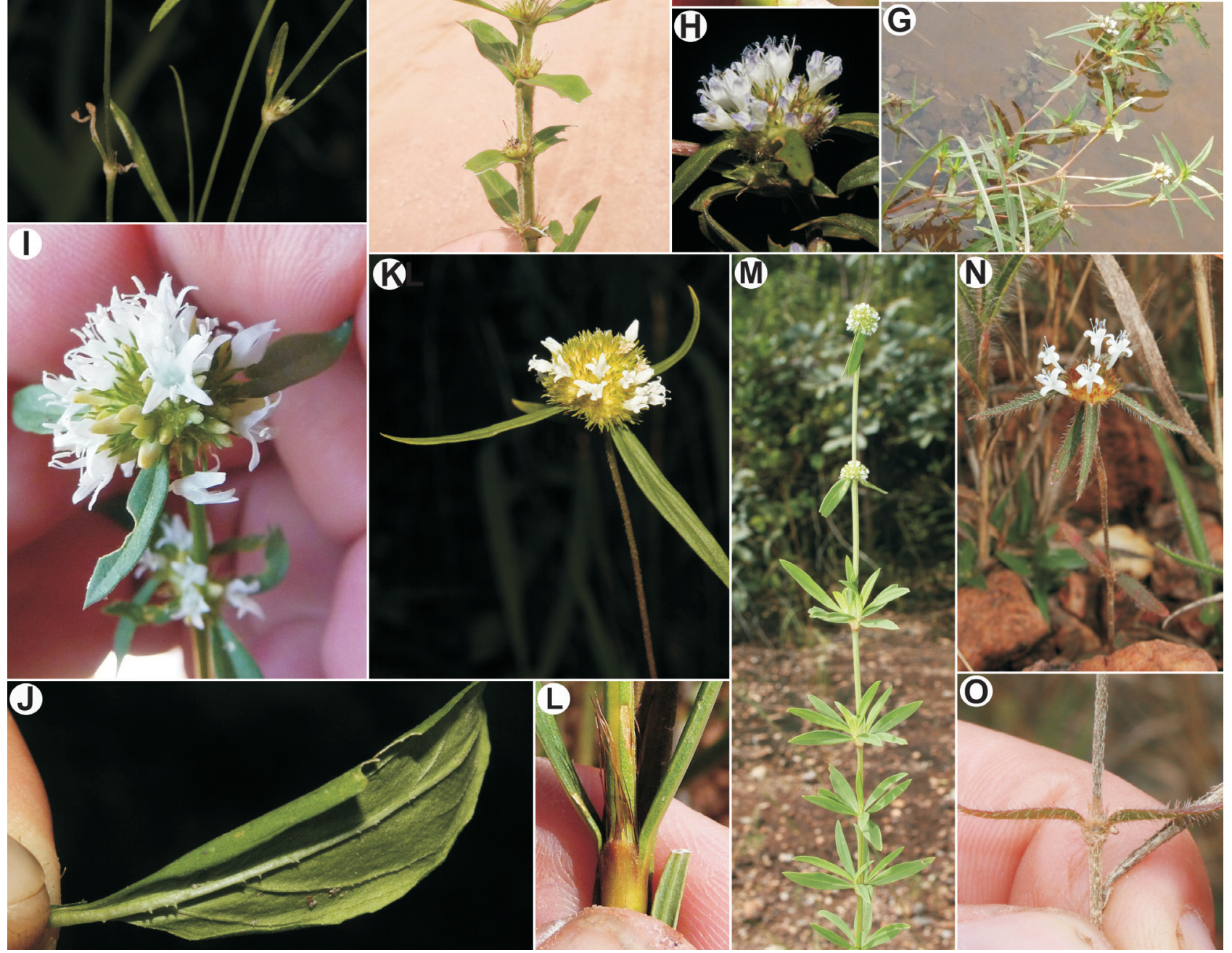

Figura 6. Fotografías de especies de Borreria. A. B. ocymoides (Burm. f.) DC.: hábito. B. B. palustris (Cham. \& Schltdl.) Bacigalupo \& E.L. Cabral: hábito. C. B. poaya (A. St.-Hil.) DC.: rama florífera. D. Borreria prostrata (Aubl.) Miq.: rama florífera. E-F. B. pulchristipula (Bremek.) Bacigalupo \& E.L. Cabral: E. Rama florífera. F. Flor con estigma bífido. G-H. B. scabiosoides Cham. \& Schltdl.: G. Hábito acuático. H. Inflorescencia apical. I-J. B. spinosa (L.) Cham. \& Schltdl.: I. Inflorescencia apical. J. Envés de hoja con papilas. K-L. B. tenella (Kunth) Cham. \& Schltdl.: K. Inflorescencia apical. L. Vaina estipular. M. B. verticillata (L.) G. Mey.: hábito. N-O. B. wunschmannii K. Schum.: N. Hábito. O. Nudo con vaina estipular y hojas opuestas. Créditos fotográficos. A: E.B. Souza; B-C, E-F, G, I, L-O: R.M. Salas; D, H, J-K: D. Cardoso. 


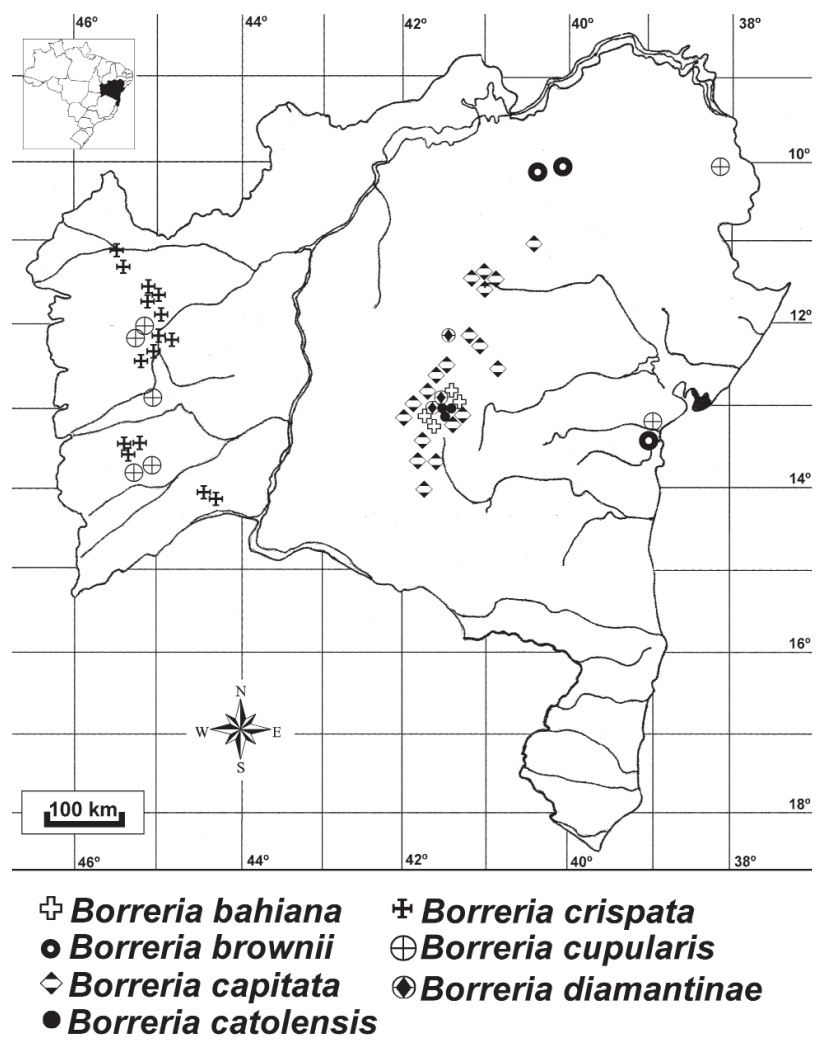

Figura 7. Distribución de las especies en Bahia (de B. bahiana a B. diamantinae).

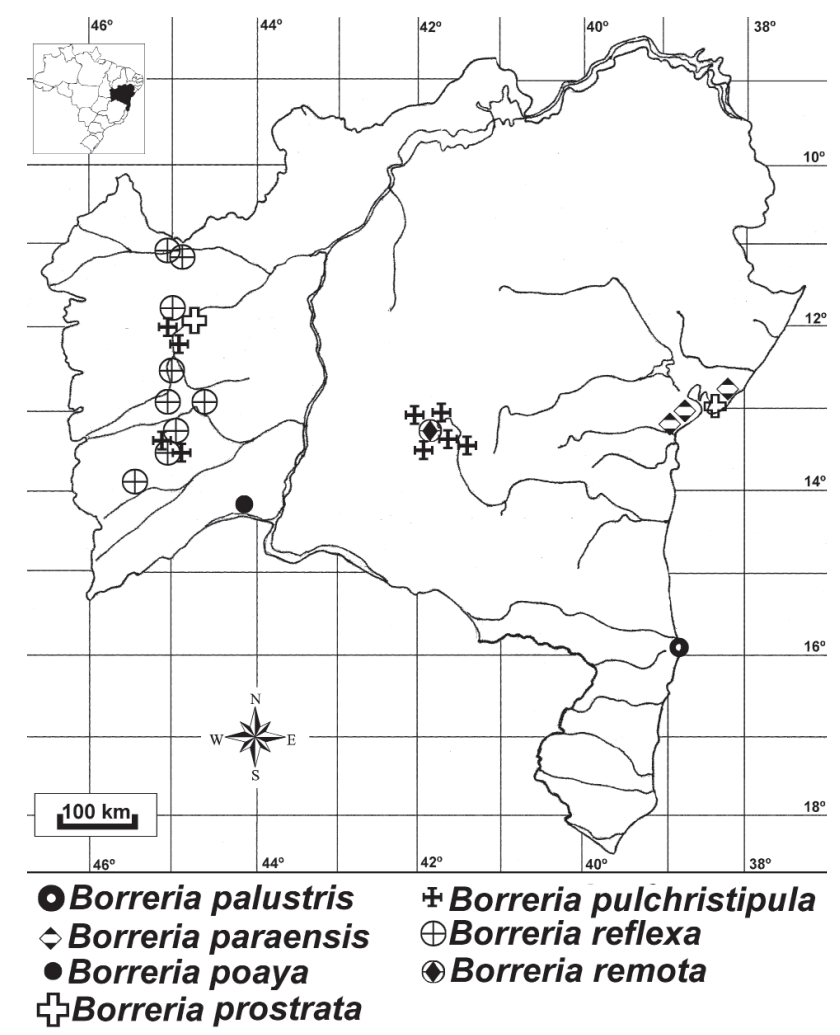

Figura 9. Distribución de las especies en Bahia (de B. palustris a B. remota).

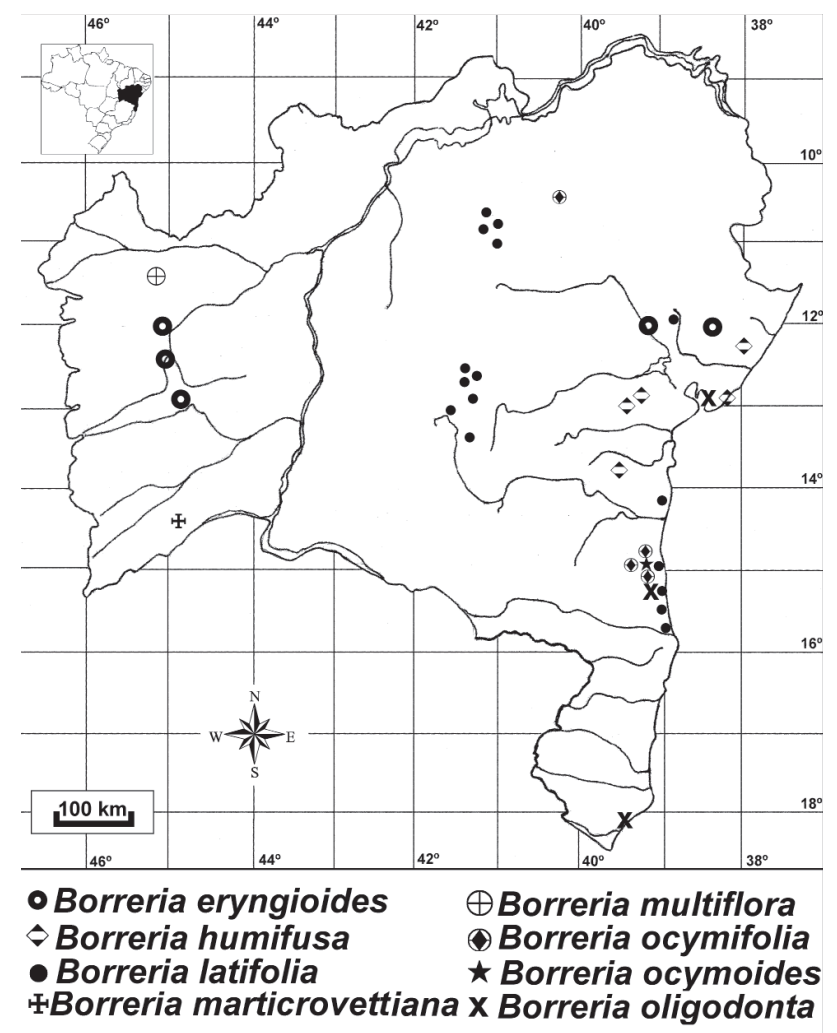

Figura 8. Distribución de las especies en Bahia (de B. eryngiodes a B. oligodonta).

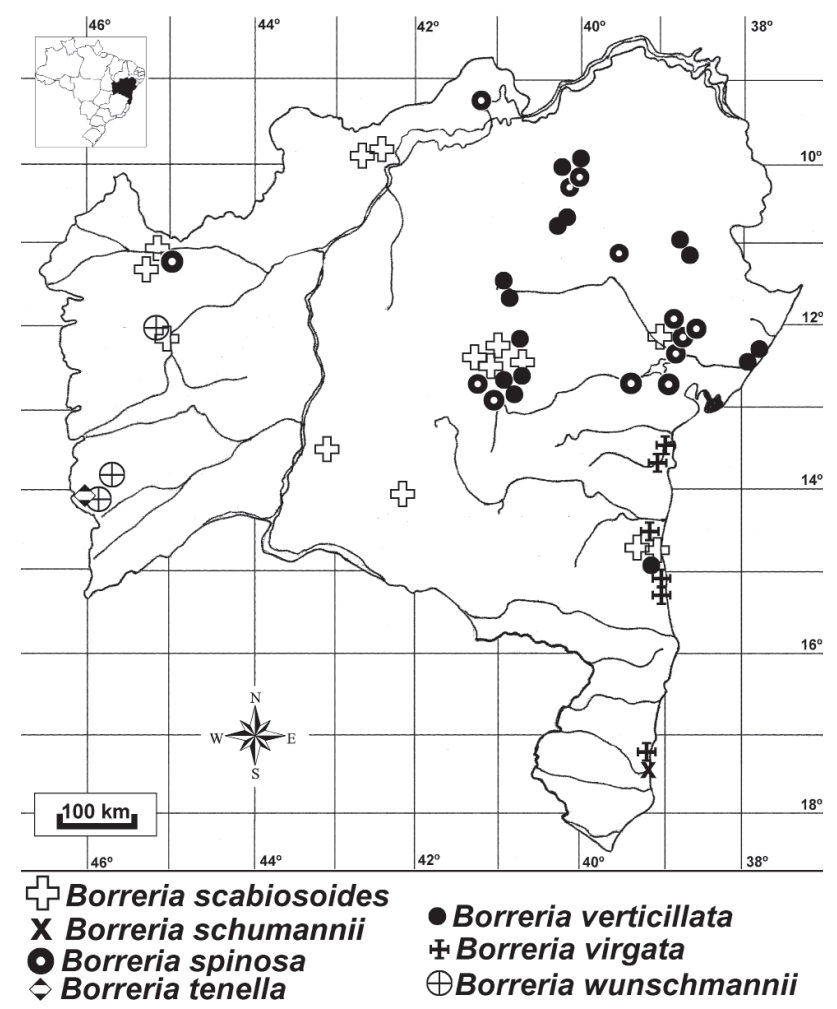

Figura 10. Distribución de las especies en Bahia (de B. scabiosoides a B. wunschmannii). 
Spermacoce virgata Link ex Roem. \& Schult., Sys. Veg. 3: 281. 1818.). Spermacoce neovirgata Govaerts, World Checkl. Seed Pl. 2(1): 18. 1996. Tipo: Brasil, Rio de Janeiro, F. Sellow $s / n$ (Holotipo B $\dagger$; Isotipos desconocidos). Neotipo Brasil, Bahia, Cairu, 1329'59'S, 39²'37'W, 15/ VIII/2008, L.P. Queiroz, E.L. Cabral, D. Cardoso, E.R. Souza \& S. Ferreira 13748 (HUEFS!, CTES!).

Fig. 4 A-H y 10

Se distribuye en British Guiana y Brasil (Bahia y Rio de Janeiro). Habita preferencialmente en Mata Atlántica, en suelos arenosos de la Restinga. Florece y fructifica de febrero a agosto.

En el protólogo de Borreria virgata es mencionado un solo ejemplar, el que fue destruído en el herbario B, y después de no hallar copias en los herbarios, se elige el neotipo.

Esta especie fue erróneamente sinonimizada por Schumann (1888) bajo Hemidiodia ocymifolia K. Schum. (= Borreria ocymifolia) concepto seguido sin cambios hasta la actualidad. Borreria virgata se diferencia de B. ocymifolia por tener una cápsula con ambos mericarpos dehiscentes (vs. un mericarpo indehiscente y otro parcialmente abierto en la base) y semillas ruminadas (vs. cara ventral de la semilla con surcos suaves que no llegan al dorso).

La mayoría del material de esta especie se encuentra identificado en los herbarios como B. capitata, de la cual se diferencia por tallos glabros lustrosos con ángulos pilosos, raro pubérulos (vs. pubescentes), hojas 6-12 x 0,5-1,9 cm, angostamente elíptico-lanceoladas, haz glabro, envés con nervios pilosos, no plegado-nervosas (vs. hojas 1,5-4 x 0,5-1 cm, elípticas u ovadas, pubescentes o escabriúsculas, plegado-nervosas), 7-10 lacinias estipulares (vs. 5 o 6), brácteas patentes 3-4 veces más largas que el glomérulo (vs. brácteas reflexas 1, raro 2, veces de igual longitud que el glomérulo), fruto subcilíndrico o elípsoide, 3 veces más largo que ancho (vs. fruto obovoide, 2 veces más largo que ancho). Entre los tipos del herbario de NY se encuentra un espécimen identificado por Steyermark como holotipo de B. capitata var. pakaraimensis, el análisis de éste y otros materiales de Guianas reveló que se trata de $B$. virgata.

Material seleccionado: BRASIL. Bahia: Ilhéus, s/d, Martii 599 (G, MO); Ilhéus, estrada para Itacaré, 14\%47’S, 39॰12'W, 25/V/2007, M.M. Silva-Castro et al. 1201 (HUEFS); Una, rod. BA-001, Ilhéus-Una, Km 41, 15¹1'1"S, 39॰00'59"W, VI/2007, J.G. Jardim et al. 4638 (HUEFS); Una, $35 \mathrm{~km}$ da rod. Olivença-Una, próxima a reserva a MicoLeão, ca. $2 \mathrm{~km}$ ao sul da entrada, 1/VI/1981, J.L. Hage e E.B. Santos 774 (CEPEC, HUEFS).

29. Borreria wunschmannii K. Schum. in Martius, Fl. Bras. 6 (6): 53. 1888. Spermacoce wunschmannii (K. Schum.) Kuntze, Revis. Gen. Pl. 3(2): 123. 1898. Tipo: Brasil, Tocantins, "prope Porto Real" (Porto Nacional), s.d., W.J. Burchell 8683 (Lectotipo BR!, designado por Cabral \& Bacigalupo 2005).
Borreria vulpina Standl., Publ. Field Columbian Mus., Bot. Ser. 8: 389. 1931. Spermacoce vulpina (Standl.) Govaerts, World Checkl. Seed Pl. 2: 19. 1996. Tipo: Brasil, Mato Grosso, Cuiabá, Coxipó da Ponte, III/1911, F.C. Hoehnne 2802 (Holotipo B†; Lectotipo SP!, aquí designado). Syn. nov.

Fig. 4I-P; 6N-O y 10.

Habita en Bolivia (Santa Cruz) y Brasil (Bahia, Goiás, Maranhão, Mato Grosso, Pará, São Paulo, Tocantins). Es una especie heliófita propia del bioma Cerrado, en suelos rocosos. En Bahia, existen escasas colecciones de la región suroeste del estado. Florece y fructifica de enero a junio.

Material seleccionado: BRASIL. Bahia: $7 \mathrm{~km} \mathrm{~S}$ of the rio Piau, ca. $150 \mathrm{~km} \mathrm{SW}$ of Barreiras, $850 \mathrm{~m}, 3 / \mathrm{IV} / 1966$, H.S.

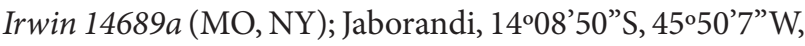
18/V/2001, F. França et al. 3711 (HUEFS); Barreiras, BR-020 en direção a Luis Eduardo Magalhães, a $20 \mathrm{~km}$ de Barreiras, $12^{\circ} 06^{\prime} 42^{\prime \prime} S, 45^{\circ} 09^{\prime} 47^{\prime \prime} \mathrm{W}, 581 \mathrm{~m}, 12 / \mathrm{IV} / 2005$, E.B. Souza et al. 1144 (HUEFS).

\section{Agradecimientos}

A Luciano Paganucci de Queiroz por la invitación a compartir los viajes de colección en Brasil; a Myndel Botanica Fundation por el financiamiento de los viajes de colección; a los curadores de los herbarios citados. A los revisores quienes han contribuido a mejorar la versión final de este trabajo.

\section{Referencias bibliográficas}

Bacigalupo, N.M. 1972. Observaciones sobre algunas especies de los géneros Spermacoce L. y Spermacoceodes O. K. (Rubiaceae). Darwiniana 17: 341-357.

Bacigalupo, N.M. \& Cabral, E.L. 1996. Infrageneric classification of Borreria (Rubiaceae-Spermacoceae) on the basis of American species. In: E. Robbrecht; C. Puff \& E. Smets (Eds). Second International Rubiaceae Conference Proceedings, Meise 1995. Opera Botanica Belgica 7: 297-308.

Bacigalupo, N.M. \& Cabral, E.L. 1998. Nota sobre dos especies de Borreria (Rubiaceae: Spermacoceae). Hickenia 2(56): 261-266.

Bacigalupo, N.M. \& Cabral, E.L. 1999. Revisión de las especies americanas del género Diodia. Darwiniana 37: 153-165.

Bacigalupo, N.M. \& E.L. Cabral. 2007. Borreria. Pp. 261, 439, 441. In Wanderley, M.G.L.; Shepherd, G.J.; Melhem, T.S. \& Giuliett, A.M. (Eds). Flora fanerogâmica do Estado de São Paulo. V.5. São Paulo, FAPESP e Instituto de Botânica.

Bacigalupo, N.M.; Cabral E.L. \& Cabaña Fader, A.A. 2010. Spermacoce spiralis, a new name for Diodia assurgens (Rubiaceae). Plant Ecology and Evolution 143: 100-104.

Brako, L. \& Zarucchi, J.L. 1993. Catalogue of the flowering plants and gymnosperms of Peru. Monographs in Systematic Botany from the Missouri Botanical Garden 45: i-xl, 1-1286.

Cabral, E.L. \& Bacigalupo, N.M. 1996. Revision of Borreria sect. Pseudodiodia. In: Robbrecht, E.; Puff, C. \& Smets, E. (Eds). Second International Rubiaceae Conference Proceedings, Meise 1995. Opera Botanica Belgica 7: 309-317.

Cabral, E.L. \& Bacigalupo, N.M. 2000. Novedades taxonômicas em Galianthe y Borreria (Rubiaceae-Spermacoceae). Bonplandia 10(14): 119-128

Cabral, E.L. \& Bacigalupo, N.M. 2005. Novelties in Spermacoceae (Rubiaceae) from Bolivia and Paraguay. Brittonia 57: 129-140. 
Cabral, E.L. \& R.M. Salas. 2010. Borreria. Pp. 1546-1549. In: Forzza, R.C. et al. Catálogo de Plantas e Fungos do Brasil 2. Rio de Janeiro, Instituto de Pesquisas Jardim Botanico do Rio de Janeiro.

Cabral, E.L.; Cabaña Fader, A.A. \& Bacigalupo, N.M. 2010. A new species of Spermacoce s.str. (Spermacoceae, Rubiaceae) from Eastern Brazil. Plant Ecology and Evolution 143(2): 1-6.

Candolle, A.P. 1830. Borreria. Pp. 540-552. In: Prodromus... V.4. Paris, Treuttel \& Würtz.

Chamisso, L.A. \& Schlechtendal, D.F.L. 1828. De plantis in expeditione speculatoria Romanzoffiana observatis. Linnaea 3(4): 338-366.

Chaw, S.M. \& Peng, C.I. 1987. Remarks on the species of Spermacoceae (Rubiaceae) of Taiwan. Journal of the Taiwan Museum 40: 71-83.

Delprete, P.G. \& Kirkbride, J.H. Jr. 2008. Clarification of Borreria gymnocephala, Diodia gymnocephala, Diodia schumannii, Borreria flavovirens, and Spermacoce schumannii (Rubiaceae). Journal of the Botanical Research Institute of Texas 2(1): 305-308.

Delprete, P.G. 2010. Spermacoce. In: P.G. Delprete. Rubiaceae, Flora dos Estados de Goiás e Tocantins (J.A.Rizzo, Coord.) 40 (2): géneros S-W, 1153-1309. Goiânia, Goiás.

Dessein, S. 2003. Systematic studies in the Spermacoceae (Rubiaceae). Doctoral Thesis. Katholieke Universiteit Leuven.

Giulietti, A.M.; Queiroz, L.P.; Silva, T.R.S.; França, F.; Guedes, M.L. \& Amorim, A.M. 2006. Flora de Bahia. Sitientibus série Ciencias Biológicas 6(3): 169-173.

Holmgren, P.N.; Holmgren, H. \& Barnett, L.C. 1990. Index herbariorum. Part I. The herbaria of the world. 8 ed. New York, New York Botanical Garden Press.

Fawcet, W. \& Rendle, A.B. 1936. Rubiaceae. Pp. 1-132. In: Flora of Jamaica 7(5). London, Oxford University Press.

Funk, V.A.; Hollowell, T.; Berry, P.; Kelloff, C. \& Alexander, S.N. 2007. Checklist of the plants of the Guiana Shield (Venezuela: Amazonas, Bolivar, Delta Amacuro; Guyana, Surinam, French Guiana). Contributions from the United States National Herbarium 55: 1-584.
Lacerda, A.L.S. 2003. Fluxos de emergência e banco de sementes de plantas daninhas em sistemas de semeaduras diretas e convencional e curvas de dose-resposta ao glyphosate. Piracicaba, Escuela Superior de Agricultura Luiz de Queiroz.

Linneo, C. 1762. Spermacoce spinosa. P. 148. In: Species plantarum. v. 1. London, Holmiae.

Liogier, A.H. 1962. Rubiaceae. Pp. 13-146. In: Flora de Cuba. v. 5. San José, Universidad de Puerto Rico.

Liogier, A.H. 1997. Rubiaceae. Pp. 65-175. In: Descriptive Flora of Puerto Rico and adjacent islands. v. 5. San José, Universidad de Puerto Rico. Martius, K.F.P. 1841. [Borreria humifusa] Rubiaceae. Flora 24(2): 68-83. Pott, A. \& Pott, V.J. 1994. Plantas do Pantanal. Brasília, Embrapa-SPI.

Rendle, A.B. 1934. Linnean species of Spermacoce. Journal of Botany 72: 329-333.

Schumann, K. 1888. Tribu Spermacoceae. Pp. 5-102. In: Martius, K.F.P. (Ed.). Flora brasiliensis 6(6). Leipzig, Fleischer.

Steyermark, J. 1972. Borreria capitata var. tenella. In: Botany of the Guayana Highland - Part IX. Memoirs of the New York Botanical Garden 23: $823-825$.

Steyermark, J.A. 1974. Tribu XVIII. Spermacoceae. Pp. 1834-1978. In: Laser, T. (Ed.). Flora de Venezuela 9(3). Caracaas, Instituto Botánico, Dirección de Recursos Naturales Renovables, Ministerio de Agricultura y Cria.

Taylor, C.M. \& Steyermark, J.A. 2004. Rubiaceae. Pp. 524-536. In: Steyermark, J.A.; Berry, P.E.; Yatskievych, K. \& Holst, B.K. (Eds). Flora of the Venezuelan Guayana. v. 8. Saint Louis, Missouri Botanical Garden Press.

Verdcourt, B. 1976. Rubiaceae (part 1) - Spermacoceae. Pp 339-374. In: Polhill, R.M. (Ed.). Flora of Tropical East Africa. London, Whitefriars Press.

Zappi, D.C.; Lucas, E.; Stannard, B.L.; Lughadha N.I.C.; Pirani, J.R.; Queiroz, L.P.; Atkins, S.; Hind, D.J.N.; Giulietti A.M.; Harley R.M. \& Carvalho A.M. 2003. Lista das plantas vasculares de Catolés, Chapada Diamantina, Bahia, Brasil. Boletim de Botânica da Universidade de São Paulo 21: 345-398. 